\title{
1 Group-wise Consistent Cortical Parcellation Based on Connectional Profiles
}

3 Tuo Zhang ${ }^{1,2}$, Dajiang Zhu ${ }^{2}$, Xi Jiang ${ }^{2}$, Shu Zhang ${ }^{2}$, Zhifeng Kou ${ }^{3}$, Lei Guo ${ }^{1}$, Tianming Liu ${ }^{2}$

$5 \quad{ }^{1}$ School of Automationand Brain Decoding Research Center, Northwestern Polytechnical

6 University, Xi'an, China; ${ }^{2}$ Cortical Architecture Imaging and Discovery Lab, Department of

7 Computer Science and Bioimaging Research Center, University of Georgia, Athens, GA,US;

$8{ }^{3}$ Departments of Biomedical Engineering and Radiology, Wayne State University, Detroit, MI,

\section{ABSTRACT}

12 For decades, seeking common, consistent and corresponding anatomical/functional regions

13 across individual brains via cortical parcellation has been a longstanding challenging problem. In

14 our opinion, two major barriers to solve this problem are determining meaningful cortical

15 boundaries that segregate homogeneous regions and establishing correspondences among

16 parcellated regions of multiple brains. To establish a corresponding system across subjects, we

17 recentlydeveloped the Dense Individualized and Common Connectivity-based Cortical

18 Landmarks (DICCCOL) system whichpossesses group-wise consistent white matter fiber

19 connection patterns across individuals and thus provides a dense map of corresponding cortical

20 landmarks. Despite this useful property, however, the DICCCOL landmarksare still far from

21 covering the whole cerebral cortex and do not provide clear structural/functional cortical

22 boundaries. To address the above limitation while leveraging the advantage of DICCCOL, in this 
1 paper, we present a novel approach for group-wise consistent parcellation of the cerebral cortex

2 via a hierarchical scheme. In each hierarchical level, DICCCOLsare used as corresponding 3 samples to automatically determine the cluster numberso that other cortical surface vertices

4 areiteratively classified into corresponding clusters across subjects within a group-wise

5 classification framework. Experimental results showed that this approach can achieve consistent

6 fine-granularity cortical parcellation with intrinsically-established structural correspondences

7 across individual brains. Besides, comparisons with resting-state and task-based fMRI datasets

8 demonstrated that the group-wise parcellation boundaries segregate functionallyhomogeneous 9 areas.

11 Keywords: Cortical parcellation, connectivity, group-wise,dMRI

\section{INTRODUCTION}

14 Identification of common and corresponding anatomical/functional cortical regions across

15 different human brainsvia cortical parcellation has been a longstanding challenge and thus has

16 attracted tremendous efforts from the neuroimaging and brain mapping fields (e.g., Thompson

17 and Toga, 1996; Shen and Davatzikos, 2002; Johansen-Berg et al., 2004; Corouge et al., 2004;

18 Fischl, et al., 1999; Cohen et al., 2008; Blumensath, et al., 2013). Current cortical parcellation

19 methodologies can be broadly divided into three categories based on the features including 20 morphological, connectional and functional features.The first group of methodologies

21 decomposes the cortex into a collection of regions of interest (ROIs) based on an atlas or

22 morphological features. For example, many such works project different brain templates/atlases

23 onto the cortex to achieve parcellation via image registration methods (e.g., Thompson and Toga, 
1 1996; Shen and Davatzikos, 2002; Fischl et al., 2004; Desikan et al., 2006; Gong et al., 2009).

2 Other works are in favor of developing morphological features (e.g., Fischl et al., 1999;

3 Lohmann and von Cramon, 2000; Rettmann, et al., 2002; Yang and Kruggel, 2008; Zhang et al.,

4 2009; Li et al., 2010a) based on the folding pattern of cortical surface, so that vertices on surface

5 with homogeneous features will be grouped into the same parcel (e.g., Zhang et al., 2009; Li et

6 al., 2010a). The second group of methodologies focus on using properties of white matter fibers

7 (e.g., Behrens et al., 2003; Anwander et al., 2007; Hagmann et al., 2007; Klein et al., 2007;

8 Tomassini et al., 2007; Behrens et al., 2008; Perrin et al., 2008; Beckmann et al., 2009; Honey et

9 al., 2009; Roca et al., 2009; Clarkson et al., 2010; Roca et al., 2010; Zhang et al., 2010; Bastiani

10 et al, 2012; Wang et al., 2013), which are usually derived from diffusion magnetic resonance

11 imaging (dMRI) data. Most of those works were in favor of using fiber bundles estimated from

12 dMRI data as connectivity of brain network and then cortical locations with similar connectivity

13 patterns were grouped into the same region. Importantly, the foundation of those works is the

14 belief that the connectivity pattern of a brain largely determines its function (Passingham et al.,

15 2002). Therefore, functional interpretation based on parcellation results were widely conducted

16 in those works and the results were promising (e.g., Behrens et al., 2003; Johansen-Berg et al.,

17 2004; Beckmann et al., 2009). Instead of seeking help from structural information, the third

18 group of methods devoted effort in using functional features to parcellate the cortex (e.g., Cordes

19 et al., 2000; Hampson et al., 2002; Beckmann et al., 2005; Cohen et al., 2008; van den Heuvel et

20 al., 2008; Nelson et al., 2010; Blumensath, et al., 2013). The important advantage of those works

21 is the easier functional interpretation of the parcellation.

23 Despite remarkable progresses made in those works, however, consistent fine-granularity 
1 parcellation of the whole cortex is still very difficult due to the complexity and variability of the

2 cerebral cortex (Zhu, et al., 2012b). In particular, methods in most previous works were

3 developed on an individual basis and it has been difficult in establishing correspondences

4 between finely-parcellated cortical regions across individuals(Zhu, et al., 2012b). Many efforts

5 have been devoted to establish cross-subject structural/functional correspondence (Corouge et al.,

6 2004; Shen, et al., 2013) by either warping an atlas or developing group-wise parcellation.

7 However, they still have difficulty in providing a stable and consistent parcellation-based cortical

8 reference system, onto which relationship between structure and function (Passingham, et al.,

9 2002; Honey et al., 2009) can be explored. For example, because of high variability across

10 subjects, functional homogeneity based on cortical parcellation result across populations cannot

11 be guaranteed (Shen, et al., 2013) and the choice of cluster number is still an open question that

12 needs to be carefully addressed. Furthermore, because of the unavailability of a stable, common

13 and consistent parcellation across subjects, it also has been difficultin obtaining clear and

14 meaningful functional and structural interpretation of the parcellation boundaries (e.g., Fischl, et

15 al., 1999; Johansen-Berg et al., 2004; Cohen, et al., 2008). In Jbabdi, et al., 2009, the authors

16 proposed a method to cluster brain regions on the basis of their connections, with no prior

17 knowledge on the number of clusters. This method also allowed combining data from different

18 subjects. However, this work was only applied to one brain region, because it needs a couple of

19 targets in other brain regions, to which connections of the region to be segmented, are estimated

20 via probabilistic tracking methods. It seems not easy to apply this method to segment a larger

21 brain region, because more targets are needed to provide connectivity profiles to segment the

22 larger brain region. However, if a set of dense target covering the full brain is chosen, the method

23 might meet a dimensionality reduction problem. 
2 Recently, in order to establish a common and consistent brain reference system, we developed 3 and publicly released a dense map of discrete cortical landmarks named DICCCOL (dense

4 individualized and common connectivity-based cortical landmarks) (Zhu et al., 2012b), and

5 demonstrated that the DICCCOL landmarks possess group-wise consistent white matter fiber

6 connection patterns across individuals and populations. From the functional point of view, the

7 neuroscience foundation of the DICCCOL system is that each brain's cytoarchitectonic area has

8 unique underlying inputs and outputs which largely determine the functions the area performs

9 (Passingham et al., 2002), and thus group-wise consistent structural and connectional profile has

10 predictive power of brain function (Zhu et al., 2012b; Passingham et al., 2002; Honey et al., 2009;

11 Li et al., 2010b).These DICCCOLs have exhibited relatively accurate structurally and

12 functionally connectional correspondences across different brains (Zhu et al., 2012b) and

13 demonstrated the promise of the connectional fingerprint concept (Passingham et al.,

14 2002).Despite that they are still far from far from covering the whole cortex and providing clear

15 structural/functional boundaries, they provide a preferable initialization for the parcellation

16 framework developed in this paper.

18 The proposed framework in this paper is conducted via a hierarchical scheme to gradually and 19 group-wisely parcellate the cortices into consistent fine-granularity parcels.Conceptually, this

20 framework leverages the proven principles in the DICCCOL system, and advances it to whole21 cortex fine-granularity parcellation. The major novelties of the framework aresummarized as

22 three-folds. 1) The cortical parcellation is performed via a hierarchical scheme so that cortical 23 surfaces are gradually parcellated into finer-granularity parcels in deeper hierarchical 
1 level(detailed in section 2.3). This hierarchical scheme provides a spectrum of multi-scale

2 parcellations, which are more flexible and accurate since it is typically difficult to determine a

3 'correct' parcellation resolution (Blumensath, et al., 2013); 2) DICCCOLs are used as samples to

4 automatically determine the cluster numbers via an adaptive affinity propagation (AP) algorithm

5 (Wang et al., 2007) (detailed in section 2.3), and this stepfundamentally guarantees the parcel

6 correspondence across subjects; 3)The group-wise implementation of a classification

7 algorithm(section 2.4),accompanied with group-wise hidden Markov random field (HMRF)

8 smoothing factor, is used to propagate cluster labels obtained in 2) onto whole-brain vertices of

9 different subjects in a group-wise fashion, so that vertices within clustered parcels possess

10 structural homogeneity and the parcels have correspondence across subjects as well (section 2.5).

\section{METHODS}

\section{$13 \quad 2.1 \quad$ Overview}

14 The overview of our framework is illustrated and summarized in Figure 1. Here, we used one 15 corresponding parcel of five subjects as an example to intuitively illustrate how the parcel was

16 gradually divided into finer ones in the hierarchical scheme. Generally, we provided a spectrum

17 of parcellations on cortical surfaces. In each hierarchical level (columns in Figure 1(a)), parcels

18 possess correspondence across subjects. Moreover, the fiber bundles extracted from the

19 corresponding parcels possess group-wise consistent patterns (Figure 1(b)). Therefore, to achieve

20 this goal, we need to solve several key problems: 1) establishing a mapping between cortical

21 surface and white matter, so that cortical surface parcellations can be the equivalent of white

22 matter parcellations (in section 2 in Supplemental Materials); 2) automatically determining the

23 cluster numbers in a group-wise manner (in section 2.3); 3) Group-wisely clustering the entire 
1 cortical surface vertices into the corresponding clusters (in section $2.4 \& 2.5$ ).

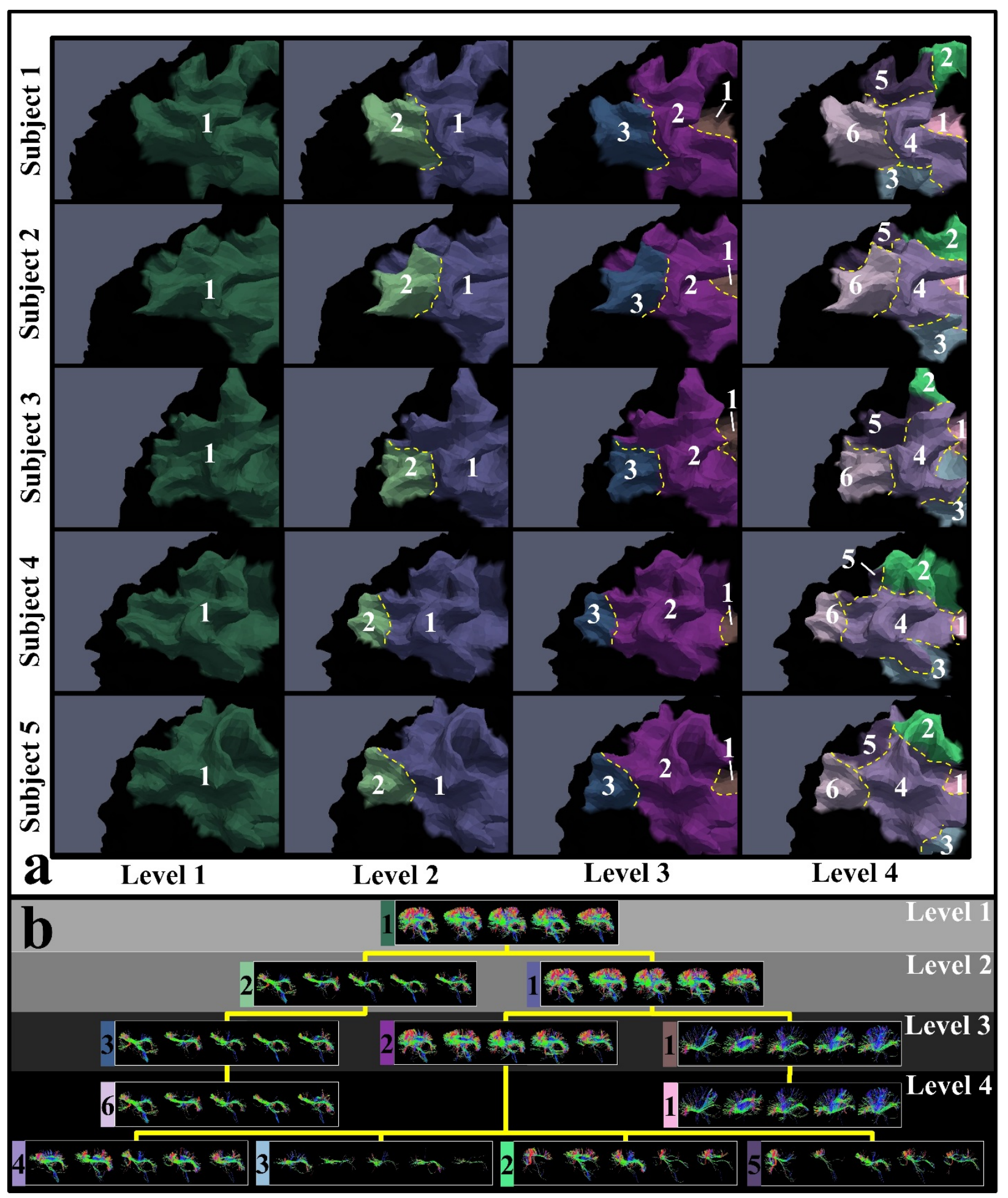

3 Figure 1. Overview of the framework and illustration of hierarchically parcellating one parcel

4 into finer ones. Five subjects are used as examples in this illustration. (a) Parcellated cortical 
1 parcels in four hierarchical levels. Corresponding parcels in each level are associated with a

2 unique number. Borders are highlighted by the yellow dash curves; (b) Fiber bundles extracted

3 from the parcels in (a). Fiber bundles extracted from the corresponding parcels of the five

4 subjects are displayed in the same box, associated with the number and color of the parcel in (a).

5 The fiber bundle boxes of all levels are organized in a hierarchical tree structure according to the

6 results in (a).

\section{$8 \quad 2.2 \quad$ Data Acquisitionand Preprocessing}

9 Dataset 1: Eight healthy young adultswere scanned in a GE 3T Signa MRI system using an 8-

10 channel head coil at the Bioimaging Research Center (BIRC) of the University of Georgia (UGA)

11 under IRB approval. The subjects participated in DTI, resting-state fMRI (rs-fMRI)scansand

12 task-fMRI scans and their datasets were used in this paper. DTI data was acquired using the

13 spatial resolution $2 \mathrm{~mm} \times 2 \mathrm{~mm} \times 2 \mathrm{~mm}$; parameters were TR $15.5 \mathrm{~s}$ and TE min-full, b-value $=1000$

14 with 30 DWI gradient directions and 3 B0 volumes acquired. All scans were aligned to the AC-

15 PC line.Rs-fMRI acquisition parameters were as follows: $64 \times 64$ matrix, 4mmslice thickness,

$16220 \mathrm{~mm}$ Field of View (FOV), 30 slices, repetition time $(\mathrm{TR})=1.5 \mathrm{~s}$, echo time $(\mathrm{TE})=25 \mathrm{~ms}$,

17 ASSET $=2$. One in-house verified paradigms fear task (Zhu et al., 2012b) was used in this paper

18 to validate the results. Task-fMRI were acquired using a T2*-weighted single shot echo planar

19 imaging (EPI) sequence and were aligned to the intercommisural line (AC - PC line);

$20 \mathrm{TE}=25 \mathrm{~ms}, \mathrm{TR}=1500 \mathrm{~ms}, 90^{\circ} \mathrm{RF}$ pulse, 30 interleaved slices, acquisition matrix $=64 \times 64$,

21 slice thickness $=4 \mathrm{~mm}, \mathrm{FOV}=240 \times 240 \mathrm{~mm}$, and ASSET factor $=2$.

23 Dataset 2 (Human Connectome Project Data, HCP for short): Diffusion MRI data of 68 
1 subjects were obtained from the Q1 release of the HCP dataset package. In brief, the imaging

2 parameters are: spin-echo EPI sequence; $\mathrm{TR}=5520 \mathrm{~ms}$; $\mathrm{TE}=89.5 \mathrm{~ms}$; flip angle=78 deg;

3 refocusing flip angle=160 deg; $\mathrm{FOV}=210 \times 180(\mathrm{RO} \times \mathrm{PE})$; matrix $=168 \times 144(\mathrm{RO} \times \mathrm{PE})$; spatial

4 resolution $=1.25 \mathrm{~mm} \times 1.25 \mathrm{~mm} \times 1.25 \mathrm{~mm}$; echo spacing $=0.78 \mathrm{~ms}$. Particularly, a full dMRI session

5 includes 6 runs (each approximately 9 minutes and 50 seconds), representing 3 different gradient

6 tables, with each table acquired once with right-to-left and left-to-right phase encoding polarities,

7 respectively. Each gradient table includes approximately 90 diffusion weighting directions plus 6

$8 b=0$ acquisitions interspersed throughout each run. Diffusion weighted data consisted of 3 shells

9 of $b=1000,2000$, and $3000 \mathrm{~s} / \mathrm{mm}^{2}$ interspersed with an approximately equal number of

10 acquisitions on each shell within each run.

12 Standard preprocessing for DTI, rs-fMRI and task-fMRI in Dataset 1 was performed in a way

13 similar to the one in Zhu et al., 2012b. Importantly, DTI space was used as intra-subject standard

14 space. For the full-set dMRI in HCP Dataset, multi-shell mode in FDT toolkit from FSL 5.0

15 (Jbabdi, et al., 2012) was used to estimate orientation distribution functions (ODFs), and DSI

16 Studio (Yeh, et al., 2013) was used to perform deterministic fiber tracking. Details about

17 preprocessing can be found in the Supplemental Materials.

19 Finally, as we parcellated the cortex via connectional profiles, we extracted fibers for each vertex

20 and adopted trace-map to depict the connectional patterns of the extracted fibers (Zhu et al.,

21 2012a). Also, based on trace-maps, a group of discrete vertices (358) distributed across the whole

22 brain, defined as DICCCOLs (Zhu et al., 2012b) were identified. As the corresponding

23 DICCCOLs across subjects share the similar connectional patterns, they were used as initial 
1 corresponding landmarks for our group-wise parcellation methods. More details about mapping

2 connectional profiles to cortical surface and establishment of DICCCOLs can be found in section

32 and 3 in the Supplemental Materials.

4

\section{$5 \quad 2.3 \quad$ Hierarchical Scheme}

6 After we associated vertices with trace-map feature vectors, the cortical parcellation problem

7 was converted into a feature clustering problem on triangular mesh space. That is to group

8 vertices with similar trace-maps into the same clusters. We also aimed to establish

9 correspondence between the vertices clusters across subjects.

11 When dealing with the clustering problem, we have to determine the cluster number. In fact, the

12 cluster number could be optimally obtained if one clustering algorithm was applied on trace-

13 maps extracted from all vertices. However, the huge number of vertices causes very heavy

14 computation load, making this optimal solution impractical. Moreover, it is our goal to determine

15 a cluster number which can be group-wisely applied onto multiple subjects, but it is difficult to

16 establish pre-defined correspondence of cortical surface vertices across subjects. Therefore, we

17 use the DICCCOLs as sampled cortical-cortex vertices, in that DICCCOL possess intrinsic

18 correspondence, that is, they exhibit common structural connectivity patterns across subjects and

19 the DICCCOLs cover the whole cortex. As a result, using DICCCOLs as samples will greatly

20 reduce the computation load (358 DICCCOLs vs. $6 \times 10^{4}$ cortical-cortex vertices). 

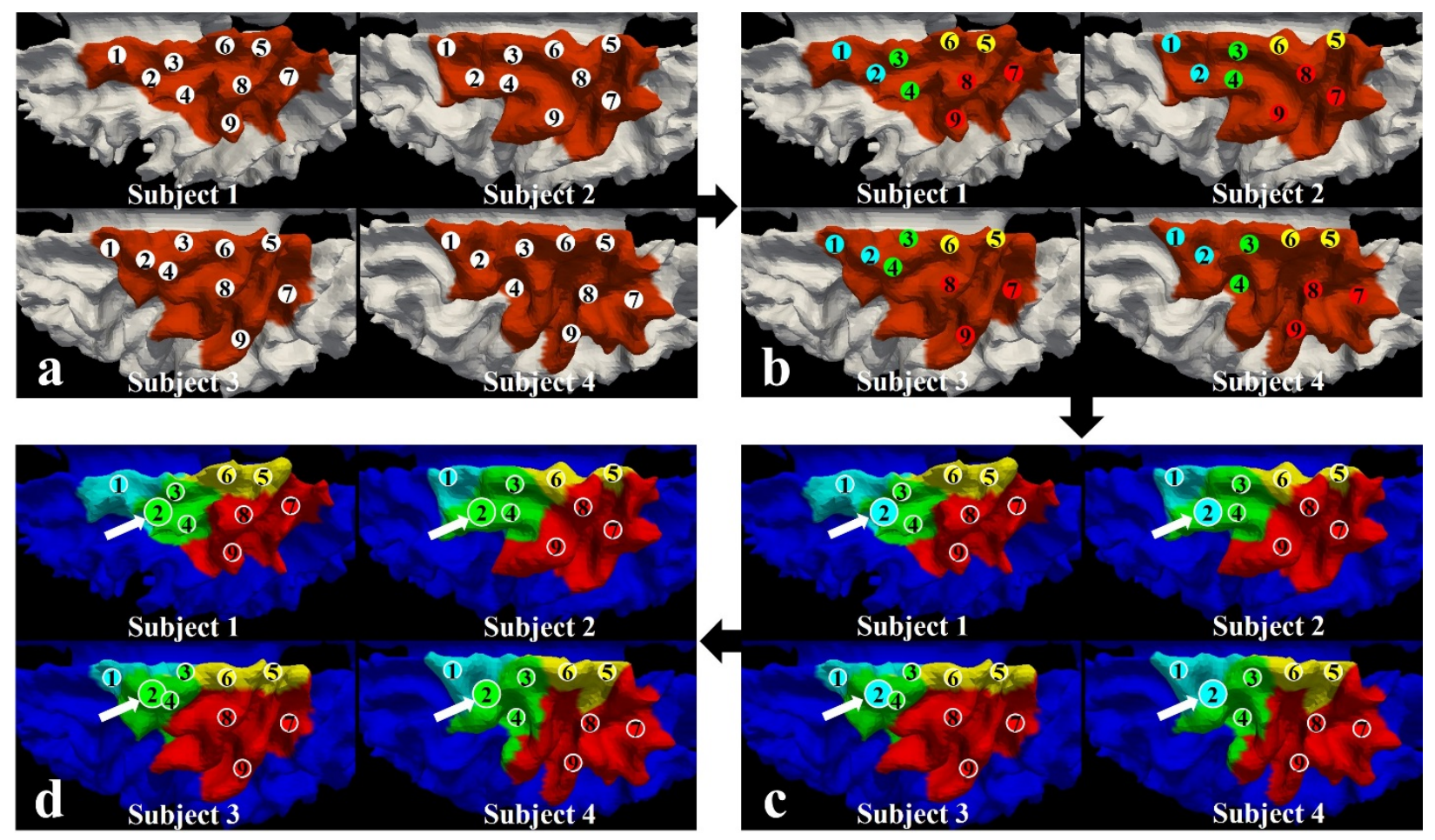

2 Figure 2. Illustration of clustering steps within one hierarchical level. Four subjects are used as

3 examples. (a) The corresponding parcels in brown color are the parcels in consideration at the

4 current level. The numbered bubbles denote nine DICCCOLs; (b) Adaptive AP clustering results

5 based on DICCCOL samples. DICCCOLs are clustered into four classes and they are of the same

6 color if they are within the same class; (c) Propagating clustering labels obtained from

7 DICCCOLs onto other vertices within the parcels in consideration via a group-wise

8 classification algorithm. Surface vertices within the same class are of the same color; (d)

9 Updating DICCCOLs classification results based on vertices propagation results. DICCCOL \#2

10 highlighted in (c) and (d) are assigned to the green-color class after updating.

12 Based on DICCCOLs, we adopted a hierarchical scheme to automatically generate a spectrum of

13 clusters.Generally, we built a divisive-style hierarchy of parcellations, through which the entire

14 surface were gradually split into finer parcels. In Figure 2(a), we used the brown color patches as 
1 an example to represent the entire surface. Once they are used in the upper level to drive

2 clustering, their cluster labels are propagated to all vertices on the surface to segment it into

3 parcels (Figure 2(d)). Then, in the next lower level, taking red parcel in Figure 2(d) for example,

4 those red DICCCOLs (DICCCOL \#7 \#9) will be used to drive clustering and the cluster labels

5 are propagated throughout the red parcel. This procedure is performed recursively.

7 Specifically, supposing the orange color parcels in Figure 2(a) are the surfaces in hierarchical

8 level \#1 to be classified. In order to group-wisely determine the cluster numbers and cluster

9 centers of these patches, sampled vertices were extracted from those. DICCCOLs are one of the

10 preferable choices to be used as sampled vertices, as they are relatively dense to cover the

11 parcels and, more importantly, corresponding DICCCOLs across subjects have similar white

12 matter connectional profiles. The outer hierarchical loop of the framework consists of the

13 following steps:

15 1. We computed the feature (trace-map in this paper) distance matrix $M_{p \times p}^{i}$ of the $\mathrm{p}$ sampled 16 vertices (DICCCOLs) for the $i^{\text {th }}$ subject of the $\mathrm{n}$ subjects. In Figure 2(a), $p=9$ and $n=4$. The

17 distance $m_{i j}$ is defined as $\left\|\vec{x}_{i}-\vec{x}_{j}\right\|^{2}$, where $\overrightarrow{\mathrm{x}}$ is the trace-map (connectional) feature. As the

$18 p$ sampled vertices have correspondences across subjects and corresponding vertices have

19 similar connectional features, we used the average $\bar{M}=\sum_{i=1}^{n} M_{p \times p}^{i}$ as the group-wise

20 distance matrix. Currently, we considered that each individual equally contributes to 21 determining the group-wise cluster number. 
1 2. We applied adaptive affinity propagation (AP) algorithm (Wang et al., 2007) on group-wise

$2 \overline{\mathrm{M}}$ to automatically classify the sampled vertices into $K$ clusters ${ }^{\text {Step* }}$ (see Figure 2(b)). Details

3 of adaptive AP in Step* can be found in Supplemental Material.

4

5 3. A group-wise classification algorithm was proposed in this work (section 2.4 and 2.5) and

6 applied to propagate classification results of the sampled vertices to the entire orange parcels

7 (see Figure 2(c)). The classification results on sampled vertices were modified according to

8 the parcellation results (see Figure 2(d)).

10 The four parcels in Figure 2(c)\&(d) comprise the hierarchical level \#2. The abovementioned

11 three steps were executed on the four parcels in a recursive manner. Each parcel was treated as

12 the one in Figure 2(a). For example, in the red parcels, we can find three DICCCOLs and they

13 are used as sampled vertices on red parcels to construct average distance matrix $\bar{M}$. Adaptive AP

14 is applied on it to classify the sampled vertices into clusters and the group-wise classification

15 algorithm is applied to propagate classification results of the sampled vertices to the entire red

16 parcel. The hierarchical scheme terminates when the total parcel numbers of the two successive

17 levels are the same, meaning that no finer-granularity parcellation can be obtained.

19 The AP algorithm (Frey and Dueck, 2007) is an automatic clustering method which takes as

20 input measures of similarity between pairs of data points. The adaptive AP was adopted rather

21 than the original AP because it is difficult to determine the value of parameter 'preference' in the

22 original AP algorithm. The parameter 'preference' roughly determines a range for cluster

23 number. The adaptive AP 'adaptively' scan the preferences to search space of the number of 
1 clusters so that it can automatically find the optimal clustering solution.

3 It is worth noting that, at the end of each hierarchical level, we updated the information that

4 which DICCCOLs locate in which corresponding parcel, because DICCCOLs are just discrete

5 samples of vertices and the clustering results based on them are only used as initial information

6 for the group-wise classification algorithm based propagation. Therefore, the final parcel

7 clustering results on the surface can possibly vary from the initialization provided by DICCCOLs.

8 As an example, DICCCOL \#2 in Figure 2(b) are grouped into the blue-color class. But after class

9 labels are propagated onto parcels in Figure 2(c), the DICCCOL \#2 locations are invaded by

10 vertices with the green-color labels, and DICCCOL \#2 class labels are updated as the green-color

11 accordingly (Figure 2(d)). Therefore, the two steps, cluster number determination based on

12 DICCCOLs (Figure 2(b)) and propagating clustering results onto parcels (Figure 2(c)), are

13 mutually updated and it is not possible that we independently construct a complete hierarchical

14 tree simply based on DICCCOLs at the very beginning and then propagate the hierarchical

15 clustering tree onto the surface accordingly. Therefore, the parcellation results via this

16 framework will remain relatively stable given a small disturbance of initialization of DICCCOLs

17 (see Supplemental Figure 3 and related context in Supplemental Materials for more details).

\section{$19 \quad 2.4 \quad$ Classification Result Propagation via a Group-wise Framework}

20 In this section, we detail how to propagate the DICCCOLs classification result in Figure 2(b)

21 onto the entire parcels in Figure 2(c). In general, this propagation problem is equivalent to a

22 parcel parcellation one, which can be formulated as a vertex clustering problem, that is, to cluster

23 cortical vertices with homogeneous connectional profiles (trace-map) into the same parcel. 
1 Supposing that we have already determined the cluster number $K$ by classifying DICCCOLs, we

2 then assume each cortical surface parcel, $k=1,2 \ldots K$, approximates a Gaussian distribution of 3 trace-maps and the entire cortex consists of $K$ Gaussian distributions with $\boldsymbol{\theta}_{k}=\left\{\vec{\mu}_{k}, \Sigma_{k}, k=\right.$ $4 \quad 1,2, \ldots K\}$, where $\vec{\mu}_{k}$ is the mean trace-map of the $k^{\text {th }}$ Gaussian distribution and $\Sigma_{k}$ is the variance 5 of trace-maps within the distribution. A straightforward rationale is illustrated in Figure3(a), in 6 which the Kendall's coefficient concordance (KCC), used to measure the similarity of the trace7 map of a vertex to those of its neighbors (similar to brain functionalregional homogeneity 8 (ReHo)in Zang et al., 2004), is mapped onto the cortical surface. More specifically, vertices 9 within 3-ring neighborhood on the cortical surface are extracted from one vertex, and then the $10 \mathrm{KCC}$ is applied on trace-maps associated with all those vertices and the value is assigned to the 11 center vertex. As the KCC measures the homogeneity of features within a region, we also use 12 regional homogeneity ( $\mathrm{ReHo}$ ) to denote it in this paper. In Figure3(a),we illustrated one ReHo 13 map. Higher values (red color) indicate that trace-map patterns at those locations are more 14 consistent. The Gaussian-like patterns visually observed in the ReHo map provide an intuitive 15 support of using Gaussian models in the group-wise classification algorithm. 


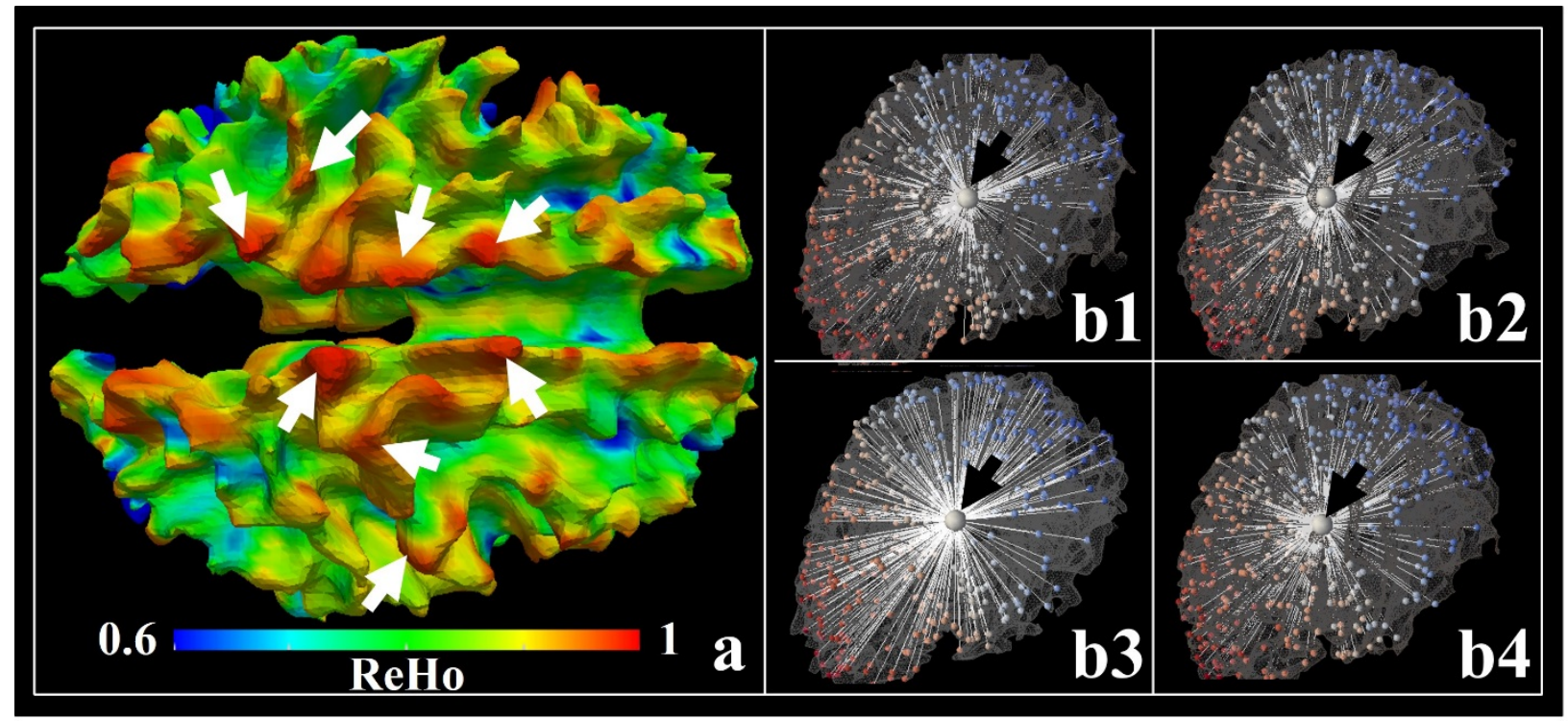

2 Figure3. (a) ReHo (regional homogeneity) map of trace-maps on the cortical surface. White 3 arrows highlight some areas with high ReHo values. (b1-b4) The four vertices (larger bubbles

4 highlighted by the black arrows) are cross-subject neighbor vertices. Smaller bubbles are

5 DICCCOLs on each subject and the white lines illustrate the Euclidean distances (In fact,

6 geodesic distances is used in the methods and Euclidean distances used in the figure is simply an

7 illustration) between the highlighted vertex and DICCCOLs. The colors of DICCCOLs represent 8 their indices.

10 Mathematically, for subject $i$ which has $M$ vertices, we used $\vec{t}_{m}$ to denote the trace-map feature of 11 vertex $\mathrm{m}$, and used $T_{i}$ to denote the collection of $M$ vertices' trace-mapfeatures, i.e., $12 T_{i}=\left\{\vec{t}_{m}, m=1,2, \ldots, \mathrm{M}\right\} \cdot p\left(y_{m k}=1\right)=\pi_{m \mathrm{k}}\left(Y=\left\{y_{m k}, m=1, \ldots, M, k=1, \ldots, K\right\}\right)$ is the prior 13 probability of assigning class labelk for vertex m, where $y_{m k}$ is a binary label and equals to 1 if 14 vertex $\mathrm{m}$ is assigned with label $k . Y$ is an $M \times K$ indicator matrix consisting of $y_{m k}$. We used $\pi / w$ 15 to denote prior/posterior probability of obtaining $Y$. As mentioned previously, a Gaussian 16 distribution $\theta_{k}=\left\{\vec{\mu}_{k}, \Sigma_{k}, k=1,2, \ldots K\right\}$ is estimated within each class and the parameters of all 
1 Gaussian distributions $\theta$ are unknown. Therefore, if we are given the class labels $Y=\left\{y_{m k}, m=\right.$

$21, \ldots, M, k=1, \ldots, K\}$, then the log-likelihood of the unknown parameters $\theta$ canbe formulated

3 based on the known trace-maps features $T_{i}$ as follows:

$$
L\left(\theta ; T_{i}, Y\right)=\sum_{m} \log \sum_{k} p\left(\vec{t}_{m} \mid y_{m k}=1, \theta\right) p\left(y_{m k}=1 \mid \theta\right)
$$

4 In other words, the Gaussian distribution $\theta_{k}$ can be estimated based on the current classification 5 results given by $\pi_{m k}(Y)$.

6

7 However, the class labels $Y$ is also unknown. To find the maximum likelihood estimate of $\theta$, we 8 devised an technique to iteratively apply two steps as follows.

9

10 Step I: Calculate the posterior probability distribution of labeling vertex $m$ as parcel $k$ under the 11 current estimate of parameters $\theta^{t}$. That is:

$$
w_{m k}^{t}=p\left(y_{m k}^{t}=1 \mid \vec{t}_{m}, \theta^{t}\right)
$$

12 Step II:Based on the probability of classification $\mathrm{w}_{\mathrm{mk}}^{\mathrm{t}}$ inStep $\boldsymbol{I}$, we maximize the likelihood

13 function in Equation (1) to estimate the Gaussian distribution parameters for each class:

$$
L\left(\theta^{t+1}\right)=\arg \max _{\theta} L\left(\theta \mid \theta^{t}, w_{m k}^{t}\right)
$$

14 More specifically, inStep II, we used $\mathrm{w}_{\mathrm{mk}}^{\mathrm{t}}$ estimated fromStep Ias the prior probability $\left(\pi_{m k}^{t}\right)$ to

15 provide classification probability, based on which parameter $\theta^{t+1}$ was estimated.After Step I, we

16 assigned label $k$ to each vertex $m$ based on $w_{m k}^{t}$. We took the 'winner-takes-all' approach for

17 each row of $w_{m k}^{t}$ to find the smallest value in each row and assign its index $k$ to the $m^{\text {th }}$ 18 vertex.Based on the classification information provided by $\pi_{m k}^{t}$, we can update the 19 parameters $\theta^{t+1}$ for all Gaussian distributions: 


$$
\begin{gathered}
\vec{\mu}_{k}^{t+1}=\frac{\sum_{m} P^{t}\left(y_{m k}^{t}=1 \mid \vec{t}_{m}\right) \vec{t}_{m}}{\sum_{m} P^{t}\left(y_{m k}^{t}=1 \mid \vec{t}_{m}\right)} \\
\Sigma_{k}^{t+1}=\frac{\sum_{m} P^{t}\left(y_{m k}^{t}=1 \mid \vec{t}_{m}\right)\left(\vec{t}_{m}-\vec{\mu}_{k}\right)^{T}\left(\vec{t}_{m}-\vec{\mu}_{k}\right)}{\sum_{m} P^{t}\left(y_{m k}^{t}=1 \mid \vec{t}_{m}\right)}
\end{gathered}
$$

1 The above classificationalgorithm is designed for a single subject $i$. In order to parcellate $N$

2 cortical surfaces simultaneously, $\theta$ is updated by considering all $N$ subjects in Step II by 3 appending $\left\{w_{i m k}, i=1, \ldots, N\right\}$ to form $w_{m k}$. No vertex correspondence between subjects is

4 needed. After obtaining the posterior probability distributions for all subjects $w_{m k}$, we split it

5 into $\left\{w_{i m k}, i=1, \ldots, N\right\}$ accordingly and assign the vertices with label $k$. We use them as a group-

6 wise prior probabilities by collecting all vertices classified into the $k^{\text {th }}$ class from all $\mathrm{N}$ subjects.

7 Then, we update the parameters $\left\{\vec{\mu}_{k}, \Sigma_{k}\right\}$ of class $\mathrm{k}$ based on the trace-maps from those $k^{\text {th }}$ class

$8 \quad$ vertices of all $N$ subjects.

\section{$10 \quad 2.5 \quad$ Group-wise Hidden Markov Random Field(HMRF) Smoothing}

11 In the above procedure, cortical surface vertices were clustered in the associated trace-map

12 feature space. So the surface vertices from distant brain areas can possibly be assigned with the

13 same class label. Therefore, we adopted the hidden Markov random field (HMRF) to prevent this

14 situationby considering that neighboring vertices typically have higher probabilityto share the 15 same cluster label (Zhang et al., 2001).

16

17 Briefly, we estimate the labels $w_{m k}^{t}=p^{t}\left(y_{m k}^{t}=1\right)$ in Step Iby Equation (6).

$$
P^{t}\left(y_{m k}^{t}=1 \mid \vec{t}_{m}\right)=\frac{\theta^{t}\left(\vec{t}_{m} ;\left\{\vec{\mu}_{k}^{t}, \Sigma_{k}^{t}\right\}\right) \cdot P^{t}\left(y_{m k}^{t}=1 \mid V_{m}\right)}{p\left(\vec{t}_{m}\right)}
$$

18 In Equation (6), $P^{t}\left(y_{m k}^{t}=1 \mid V_{m}\right)$ encodes the similarity of other vertices' labels within the 
1 neighborhood system $V_{\mathrm{m}}$ to labeling vertex $\mathrm{m}$ as class $\mathrm{k}$ and it can be written in the form:

$$
p\left(y_{m k}=1 \mid V_{m}\right) \propto \exp \left\{-\frac{1}{2} y^{T} V_{m} y\right\}
$$

2 where $y$ is the label of vertices within the neighborhood.The element $\operatorname{in} \boldsymbol{V}_{\mathrm{m}}$ is 1 if avertex is 3 within the neighborhood.

5 The neighborhood on one subject surface can be established straightforwardly by including all

6 surface vertices linked to the vertex in consideration by surface edges. In order to extend the

7 neighborhood system to a group-wise domain, we used the DICCCOLs as references and 8 computed the geodesic distance of the vertex m from subject $i$ to all DICCCOLs of the same

9 subject, so that we obtained a distance vector $\vec{g}_{m}^{i}$. Then, the neighbor vertex of $v_{m}^{i}$ on subject $j$ is

10 defined as the one with the most similar $\vec{g}_{m}^{j}$ by measuringthe Euclidean distance between the

11 distance vectors. As an example, we randomly selected a vertex from one subjecthighlighted by

12 the black arrow in Figure3(b1), and its neighboring vertices on other three subjects are shown 13 inFigure3(b2-b4), as highlighted by the black arrows.

\section{RESULTS}

\section{$16 \quad 3.1 \quad$ Structural Validation of theCortical Parcellation Results}

17 In this section, our methods will be evaluated in the structure aspects by comparing the results

18 with several other methods. We used the eight subjects from Dataset 1 to evaluate the group-

19 wise parcellation framework. The cluster number stops increasing after level 5 in the hierarchical

20 scheme and the group-wise classification algorithm converges after around 50 iterations on most

21 parcels at each level. As an example, the parcellated cortical surfaces of fiveexample subjects

22 werevisualized in the first five columns in Figure 4 (the results within the same hierarchical level 
1 are in the same column). Structural connectivity matrices based on the level-5 parcellation results

2 were also illustrated in column $\mathrm{f}$. The consistency of parcel location distributions and patterns of

3 structural connectivity matrices across subjects can be qualitativelyappreciated in Figure 4.

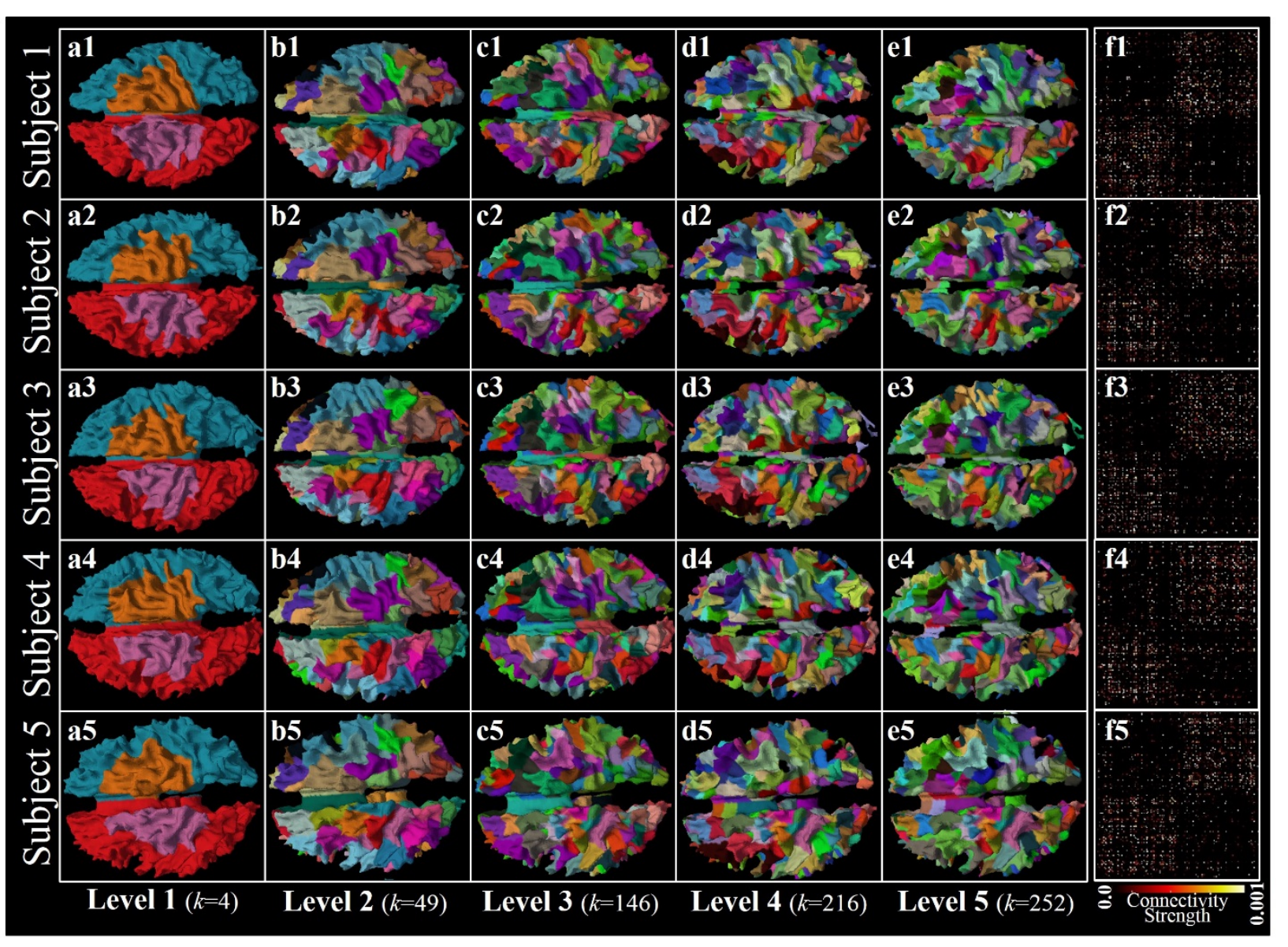

5 Figure 4. (a1-e5) Group-wise surface parcellation results of five example subjects (rows) in five

6 levels (columns). Corresponding parcels in each column share the same color. (f1-f5) Structural

7 connectivity matrices derived from the parcellation results in (e1-e5). The color bar indicates the 8 connectivity strength.

9

\section{Group-wise Results vs. Individual Based Results}

11 We also visualized the comparison between the group-wise parcellation results (level 5) with

12 those obtained from a single subject (the same subjects as those used in the group-wise 
1 framework), and the resultsare shown in Figure 5. The parcellation for a single subject is

2 accomplished by using the same framework, except that it is applied on one subject each time. In

3 order to make the individual results comparable to the group-wise results, the DICCCOL

4 clustering results from the group-wise results at each level were directly used as the initialization

5 for single subject parcellation.

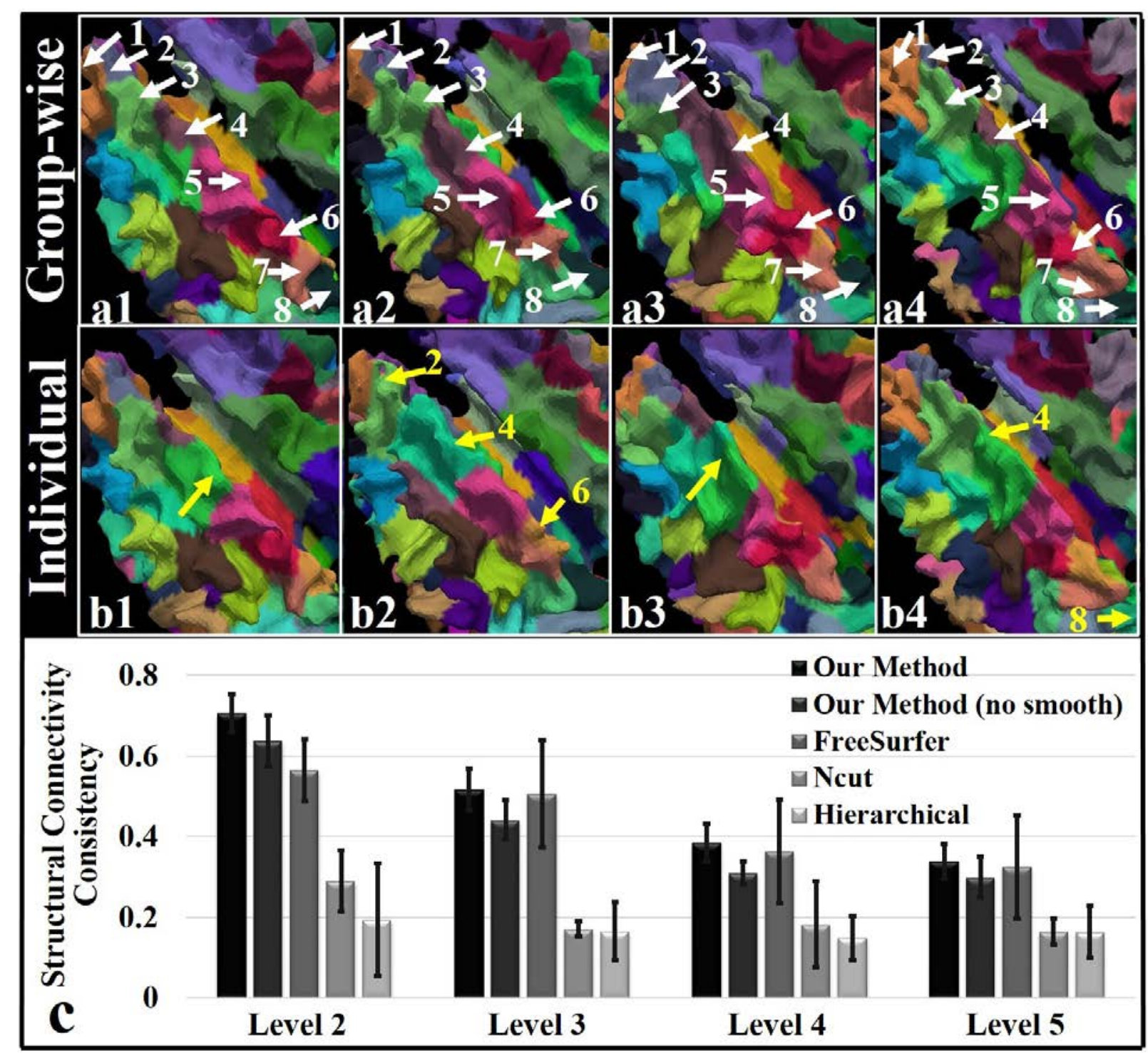

7 Figure 5. (a1-b4) Zoomed-in views of parcellation results on the left frontal lobes of four

8 example subjects via the group-wise classificationalgorithm(a1-a4) and the onefor

9 individuals(b1-b4). The white arrows highlight the corresponding parcels. The yellow arrows 
1 highlight missing parcels or invading parcels. (c) Consistency measure of structural connectivity

2 across subjects. The bins and bars show the average consistency and the standard deviation.

4 We zoomed in the left frontal lobes and identified 8 parcels in Figure 5. We can observe from 5 Figure 5(a1-a4) that the eight parcels (highlighted by the white arrows)parcellated by the group6 wise classification algorithm follow exactly the same order from the anterior frontal lobe to the 7 posterior frontal lobe on different subjects. In comparison, the parcellated parcels based on single 8 subject are much less consistent (Figure 5(b1-b4)). The yellow arrows with parcel IDs highlight 9 the missing parcels, while the ones without parcel IDs indicate some other invading parcels. This 10 consequently leads to missing or variant fiber bundles and thus further reduces the structural and 11 functional connectivity consistency across subjects.

13 Quantitatively, we compared the whole-cortex structural connectivity matrices based on the

14 level-5 group-wise/individual parcellation results. On average, the structural consistency

15 measurement via the group-wise parcellation is 0.34 , which is significantly greater than the one

$16(0.26)$ via individual parcellation $\left(p=6.57 \times 10^{-6}\right.$ by one tailed $t$-test). Similarly, functional 17 connectivity consistency was also measured based on rs-fMRI data. On average, the consistency 18 measure among functional connectivity matrices via group-wise parcellation is 0.48 , which is 19 significantly greater than the one ( 0.41$)$ via individual parcellation ( $p=0.003$ by one tailed $t$-test).

\section{HMRF Smoothing Factor}

22 In order to demonstrate the effectiveness of group-wise HMRF smoothing, we computed the 23 structural connectivities obtained by our methodswith/without group-wise HMRF 
1 smoothingbased on the parcellations on different levelsand compared the connectivity

2 consistency in Figure 5(c).Taking level-5 results for example, the consistency decreases

$3 \quad(0.30 \pm 0.05$ without smoothing vs. $0.34 \pm 0.04$ with smoothing) when group-wise HMRF

4 smoothing was removed from the method. The rationale is that two distant and disconnected

5 parcels are likely to be classified into one cluster if our method is only applied in the structural

6 feature space with no surface-based spatial smoothing constraint, and this will consequently

7 reduce the structural connectivity consistency across subjects.

\section{Comparison with Other Methods}

10 We compared the structural connectivity consistency of the parcellations derived from our group-

11 wise method at the resolution of different levels with those by the Ncut method (Von Luxburg,

12 2007) and the hierarchical method using the Ward's linkage rule (Ward, 1963), and reported the

13 results in Figure 5(c). Unlike our method that can generate correspondence of parcels (the color

14 parcels in Figure 5(a)), the Ncut and hierarchical methods, however, have difficulty in

15 establishing correspondence across subjects. We used the Dice similarity method detailed in the

16 Supplemental Materials to establish the parcel correspondence across subjects for those two

17 individual based methods. Then the structural connectivity matrices and the structural

18 connectivity matrices consistency were measured based on the corresponding parcellations.

19 Generally, our methods outperform the two methods. For example, the structural connectivity

20 consistency derived from our method is $0.34 \pm 0.04$ at level 5 , which is significantly higher than

$210.16 \pm 0.03$ and $0.16 \pm 0.06$ for the Ncut and hierarchical methods. The reason that the latter two

22 methods generate low structural consistency is that these two methods are individual based ones,

23 so that there is no intrinsic correspondence of parcels across subjects. 
2 We also compared our method with the registration method. Specifically, one subject of those

3 used in our group-wise parcellation method was defined as the 'template' subject. Then, the

4 registration method in FreeSurfer (CVS registration, Postelnicu et al., 2009, Zöllei et al., 2010)

5 was used to warp the surface of the 'template' subject to every other subject one by one. The

6 parcellation of the 'template' subject was mapped to other subjects accordingly. The parcellation

7 of the 'template' subject was originally obtained from our group-wise parcellation method, and

8 by this parcellation method every subject thus has the same number of parcels (n parcels).

9 Therefore, in the registration method, every subject also has ncorresponding warped parcels as

10 well. We measured the structural connectivity consistency of the parcellation among subjects for

11 the registration method (Figure 5(c)). On average, the consistency is $0.30 \pm 0.05$, which is lower 12 than our method $(0.34 \pm 0.04)$.

\section{$14 \quad 3.2 \quad$ Comparison BetweenCortical Parcellation and fMRI Data}

15 Although the effectiveness of our methods has been validated via several structure-based

16 measurements in section 3.1, the functional interpretation of the structural parcellation has not

17 been studied. In this section, we will use rs-fMRI and task-fMRI derived maps and clusters to 18 study if the structural parcellation also has meaningful functional interpretation. 


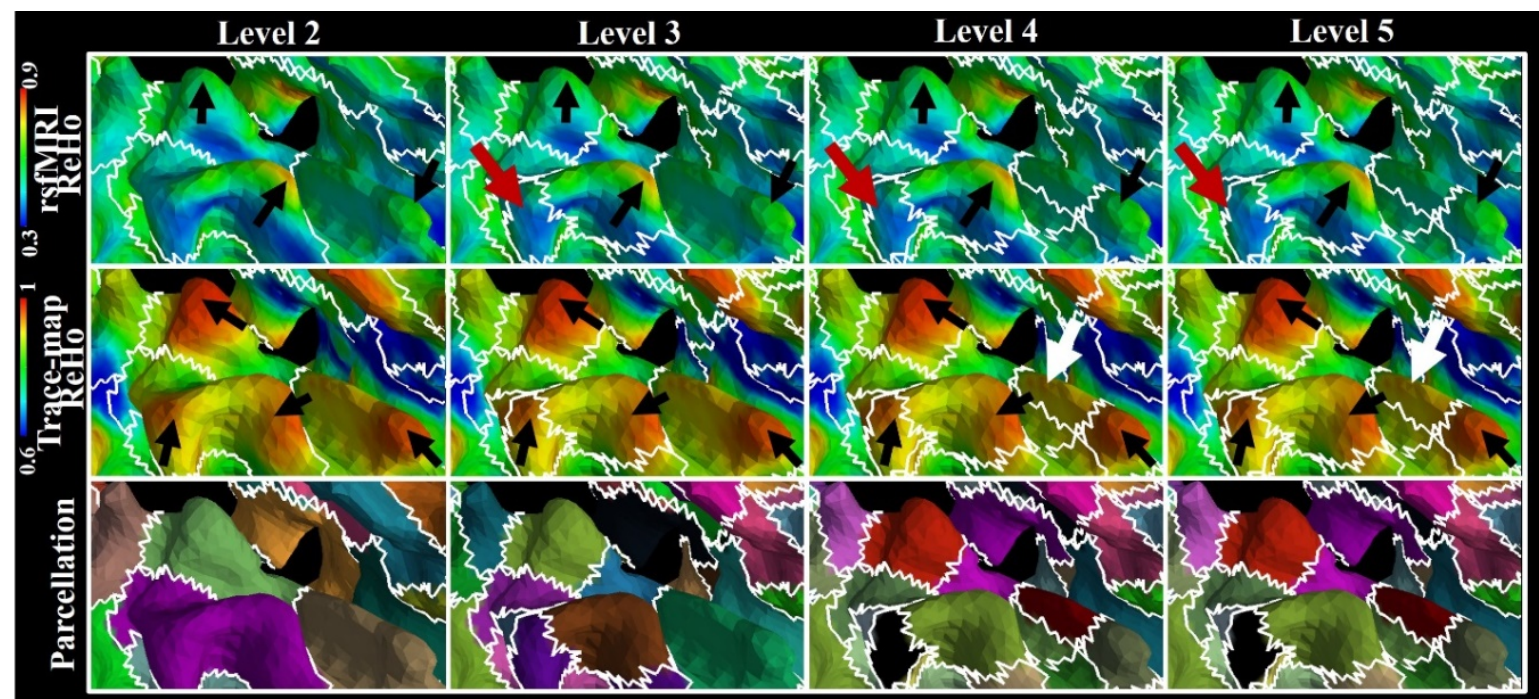

a
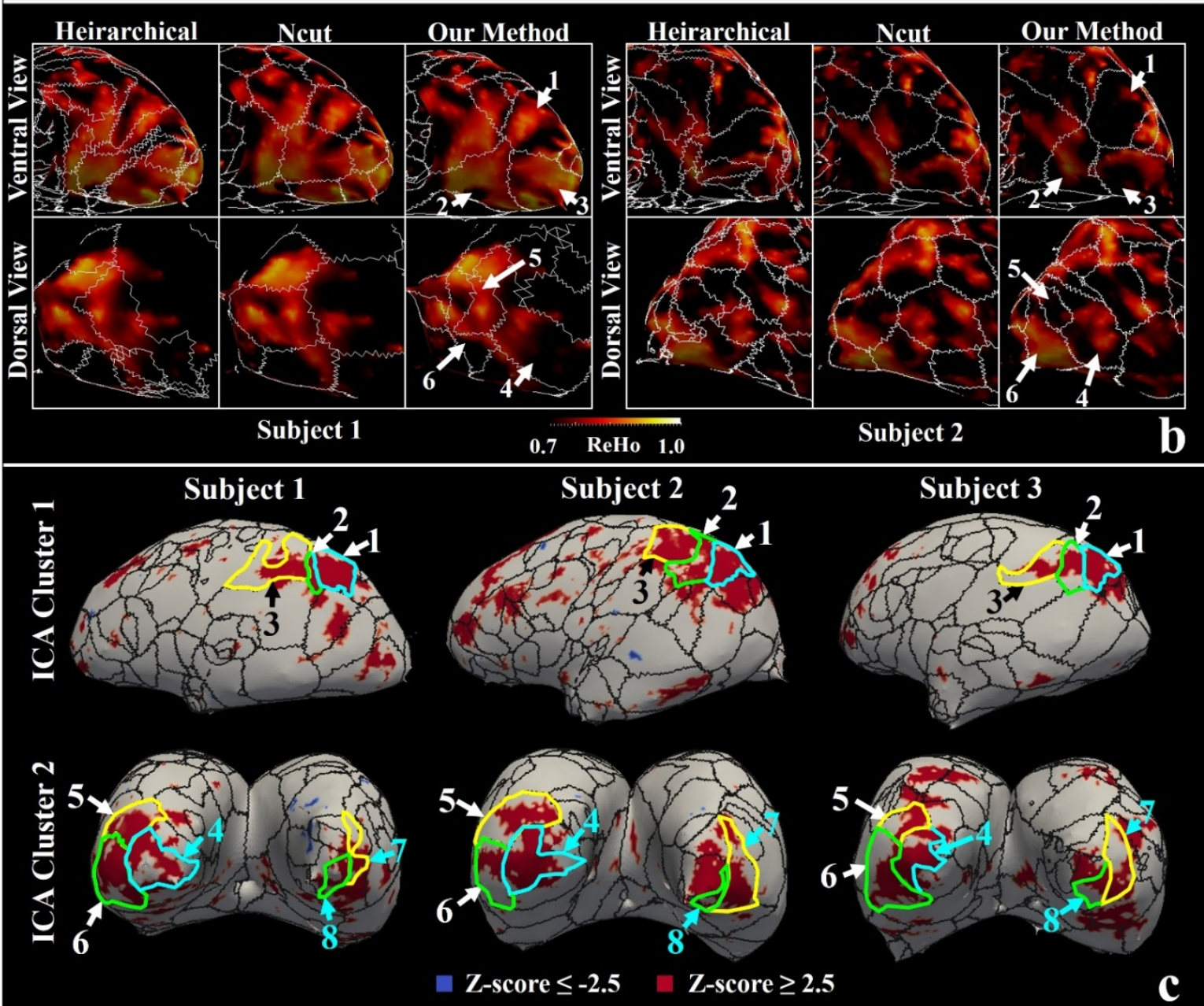

C

2 Figure 6.Comparison of structural parcellation borders and rs-fMRI derived ReHo maps. (a)

3 Joint visualization of parcellation borders (the white curves) and ReHo maps of one subject. The 
1 parcellation borders are overlaid on rs-fMRI derived ReHo map (the top row), trace-map-derived

2 ReHo map (the middle row) and parcellated surfaces (the bottom row). The interpretation of

3 arrows is referred to the main text; (b) Parcellation borders (at a resolution of 252 parcels) via

4 different parcellation methods are overlaid over ReHo maps (visual cortices) on a scale of 0.7 to

5 1. Numbered arrows indicate correspondent parcels obtained from our methods. (c) Parcellation

6 borders (at a resolution of 252 parcels) via our methods are overlaid over two ICA clusters

7 derived from rs-fMRI. Corresponding parcels, indicated by numbered arrows, are highlighted by

8 the borders with the same color.

10 Parcellation Borders Aligned with Homogeneous Maps and ICA Clusters from Rs-fMRI

\section{Data}

12 In this section, the structural parcellation boundaries were analyzed by comparison with the rs-

13 fMRI derived ReHo maps and ICA clusters. The functional correspondence based on structural 14 parcellation was also discussed.

16 Generally, ReHo assumed that voxels/vertices within a functional brain area were more

17 temporally homogeneous when this area is involved in a specific condition. Therefore, brain

18 regions with higher ReHo values suggest that a homogeneous brain activity would likely occur

19 (Liu et al., 2008), and the regions with lower ReHo values might suggest a possible boundary

20 segregating different brain regions engaged in different brain activities. We overlaid the

21 parcellation boundaries of one subject in different hierarchical levels on the ReHo maps and

22 showed them in a zoomed-in view in the first row of Figure 6(a). Corresponding ReHo maps

23 based on the trace-map (the second row) and the parcellation results (the bottom row) are also 
1 shown as reference. We can observe four structural ReHo peaks (black arrows in the second row).

2 When the algorithm runs into finer levels (\#3, \#4 and \#5), the four peaks are completely

3 separated by boundaries and no over-parcellation occurs except one hidden peak (the white

4 arrows), suggesting the effectiveness of the stopping criterion of the hierarchical scheme. As for

5 the functional ReHo derived from rs-fMRI data, the structural parcellation borders also roughly

6 match the areas with low functional ReHo values and ReHo peaks are also found within parcels

7 (the black arrows in the first row of Figure 6(a)). The average functional ReHo value obtained

8 from the vertices that the parcellation bordersof the four levels in Figure 6(a) pass by is 0.52 ,

$90.54,0.54$ and 0.55 , which are less than the overall average functional ReHo values (0.56). This

10 result suggests that the structural borders may also segregate functionally homogeneous regions.

11 It should be noted thatit is possible that no functional ReHo peaks can be found in the parcels

12 highlighted by the red arrows while structural ReHo peaks exist.

14 We also compared our method with the Ncut and hierarchical methods by overlaying different

15 parcellation borders onto the rs-fMRI ReHo maps. In Figure 6(b), we visualized the comparison

16 made on visual cortices. Based on visual observation, structural boundaries based on three

17 methods somewhat match the low functionally homogeneous regions. The Ncut borders perform

18 worse to segregate functionally homogeneous regions in the visual cortex, while the hierarchical

19 method borders are more redundant than others at the same parcellation resolution. On the

20 contrary, borders based on our methods provide preferable functional segregation performance

21 and simultaneously possess parcel correspondence (the numbered arrows in Figure 6(b)). The

22 structural correspondence may suggest a similar correspondence of functionally homogeneous

23 regions across subjects (the functionally homogeneous regions in \#2, \#3 and \#4 parcels), though 
1 functional variation across subjects can also be spotted. For example, \#1 parcels share similar

2 structural profiles but no functionally homogeneous feature can be observed in subject 2 . The

3 same situation happens on \#5 and \#6 parcels as well.

4

5 Quantitatively, we overlaid the structural borders via our method over ICA analysis derived

6 clusters in Figure 6(c). The two ICA clusters in rows may have correspondences across subjects

7 based on the component correspondence identification method introduced in the Supplemental

8 Materials (please refer to the numbered corresponding parcels the ICA clusters overlapped with).

10 Parcellations Possessing Group-wise Functional Connectivity Consistence on Rs-fMRI

\section{Data}

12 We computed the functional connectivity consistency based on the parcellations of different level.

13 Ncut method, hierarchical methodand FreeSurfer registration methodare also used for

14 comparison. Similarly, we used the Dice similarity method to establish the parcel correspondence

15 across subjects for those two individual based methods. We reported in Table 1the average

16 consistency measure of functional connectivity derived from rs-fMRI across subjects. The

17 intrinsic correspondence of parcels across subjects obtained via our methods results in much

18 better functional connectivity consistency than other ones.

20 Table 1. Average consistency measure \pm standard deviation of functional connectivity across 21 subjects.

\begin{tabular}{c|cccc}
\hline & LEVEL 2 & LEVEL 3 & LEVEL 4 & LEVEL 5 \\
\hline OUR METHOD & $0.65 \pm 0.06$ & $0.56 \pm 0.05$ & $0.52 \pm 0.05$ & $0.48 \pm 0.04$ \\
FREESURFER & $0.61 \pm 0.07$ & $0.50 \pm 0.13$ & $0.45 \pm 0.11$ & $0.42 \pm 0.10$ \\
NCUT & $0.31 \pm 0.15$ & $0.15 \pm 0.22$ & $0.21 \pm 0.26$ & $0.15 \pm 0.23$ \\
HIERARCHICAL & $0.01 \pm 0.07$ & $0.17 \pm 0.13$ & $0.09 \pm 0.13$ & $0.10 \pm 0.15$ \\
\hline
\end{tabular}


2 We showed in Figure 7 a zoomed-in view of the parcellation borders at the resolution of $49 \& 146$

3 parcels overlaid over the fear task in Dataset 1. The alignment of parcellation borders with task

4 activation cluster boundaries can be observed in Figure 7.

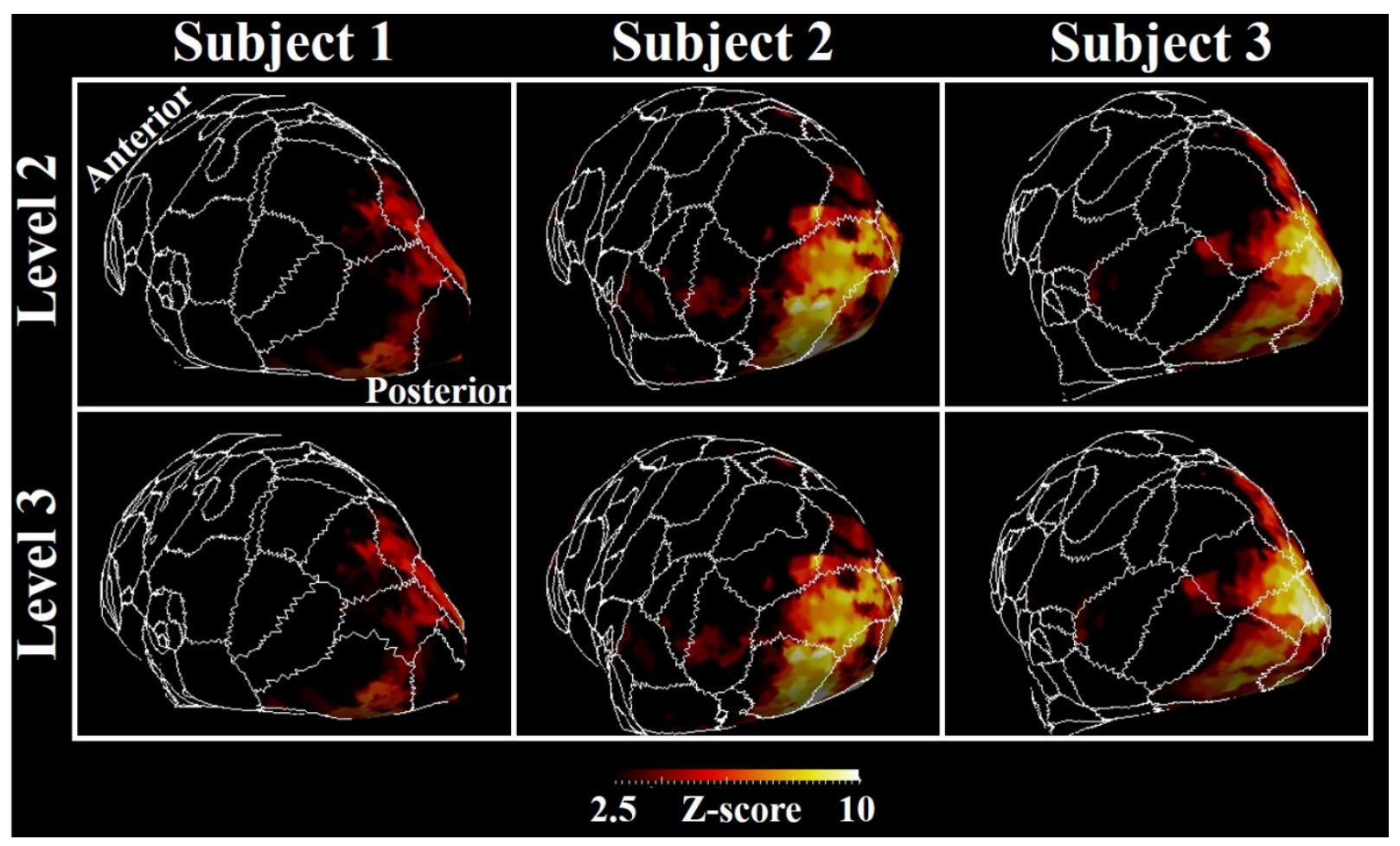

6 Figure 7. Level 2\&3 (at the resolution of 49\&146 parcels) parcellation borders overlaid over fear

7 task activation maps of three subjects' primary visual cortices. Some correspondent parcels are

8 highlighted by the dashed curves of the same color.

9

\section{$10 \quad 3.3 \quad$ Reproducibility on HCP Dataset}

11 In this section, we demonstrated the reproducibility of our methodsonan independent dataset. It

12 is noted that we used full-set (multi-shell) dMRI data from 8 HCP subjects to explore if richer

13 connectional information provided by HCPdata can produce similar parcellation results. 
1 It is straightforward that we directly applied our methods on the 8 HCP subjects to produce

2 group-wise parcellations. However, it is difficult to compare the parcellation results with Dataset

31 derived ones, as there are not overlapping subjects from the two datasets, and we have to

4 conduct inter-subjects registration to warp parcellation from Dataset 1 to HCP dataset. This is

5 subject to registration errors. Therefore, we adopted strategy \#1 as follows:

7 Strategy \#1 is using combined Dataset 1 and HCP Dataset. However, the computation load and

8 memory cost would be very heavy on 16 subjects ( 8 from Dataset 1 and 8 from HCP Dataset)

9 for the current method. Therefore, we conducted two experiments using our group-wise

10 parcellation method on two data groups: 1) the 8 subjects in Dataset 1 plus one HCP subject

11 (e.g.,subject \#i); 2) onlythe 8 subjects in HCP Dataset (including subject \#i). Then, the

12 parcellation results of the HCP subject \#i from experiments \#1 and \#2 are compared. As

13 subjects in Dataset 1 dominate the data group in experiments \#1, the parcellation on HCP

14 subject \#i encodes the group-wise information from Dataset 1. For experiments \#2, parcellation

15 on HCP subject \#i purely encodes the group-wise information from HCP Dataset. Therefore, by

16 this way, HCP subject \#i is the intermedia onefor which parcellations from Dataset 1 and $\mathbf{H C P}$

17 Dataset are compared without introducing inter-subject registration errors. The comparison

18 between the two parcellations was measured via Dice similarity. This strategy is performed on

19 every HCP subject.

21 As a comparison, we also adopted strategy \#2, a registration strategy:

22 Strategy \#2 is using the methods in FreeSurfer to register subjects in Dataset $\mathbf{1}$ to HCP subjects

23 and warp the parcellations accordingly. Then the warped parcellations were compared to those 
1 derived from HCP subjects themselves via Dice similarity measurements. This strategy is

2 subject to inter-subjects registration errors.

4 The Dice similarity measures at different resolutions of parcellation levels were reported in

5 Table 2. On average, Dice similarity is $(0.33 \pm 0.12)$ for strategy \#1, suggesting that independent

6 datasets can produce similar parcellations, and this similarity is more pronounced than using

7 registration method (Dice similarity is $0.24 \pm 0.03$ for strategy \#2). Figure 8 shows one HCP

8 subject as an example. The parcellation on it was obtained via applying our group-wise method

9 only on HCP Dataset (experiment \#2 via strategy \#1). To this subject, the parcellation

10 boundaries of one subject from Dataset 1 via registration in strategy \#2 were superimposed

11 (Figure 8(b)). The parcellation boundaries of the same HCP subject via experiment \#1 in

12 strategy \#1 were also superimposed and shown in Figure 8(a). Many matched parcellation

13 boundaries can be found in Figure 8(a) (black arrows highlighted ones), giving an intuitive

14 explanation of the reasons that Dice similarity for strategy \#1 is higher than strategy \#2.

15

16 Table 2. Average Dice similarities between parcellations via different comparison strategies.

17

\begin{tabular}{l|cccc}
\hline & LEVEL 2 & LEVEL 3 & LEVEL 4 & LEVEL 5 \\
\hline STRATEGY \#1 & $0.28 \pm 0.14$ & $0.34 \pm 0.10$ & $0.35 \pm 0.11$ & $0.35 \pm 0.11$ \\
STRATEGY \#2 & $0.25 \pm 0.06$ & $0.24 \pm 0.04$ & $0.24 \pm 0.03$ & $0.24 \pm 0.03$ \\
\hline
\end{tabular}




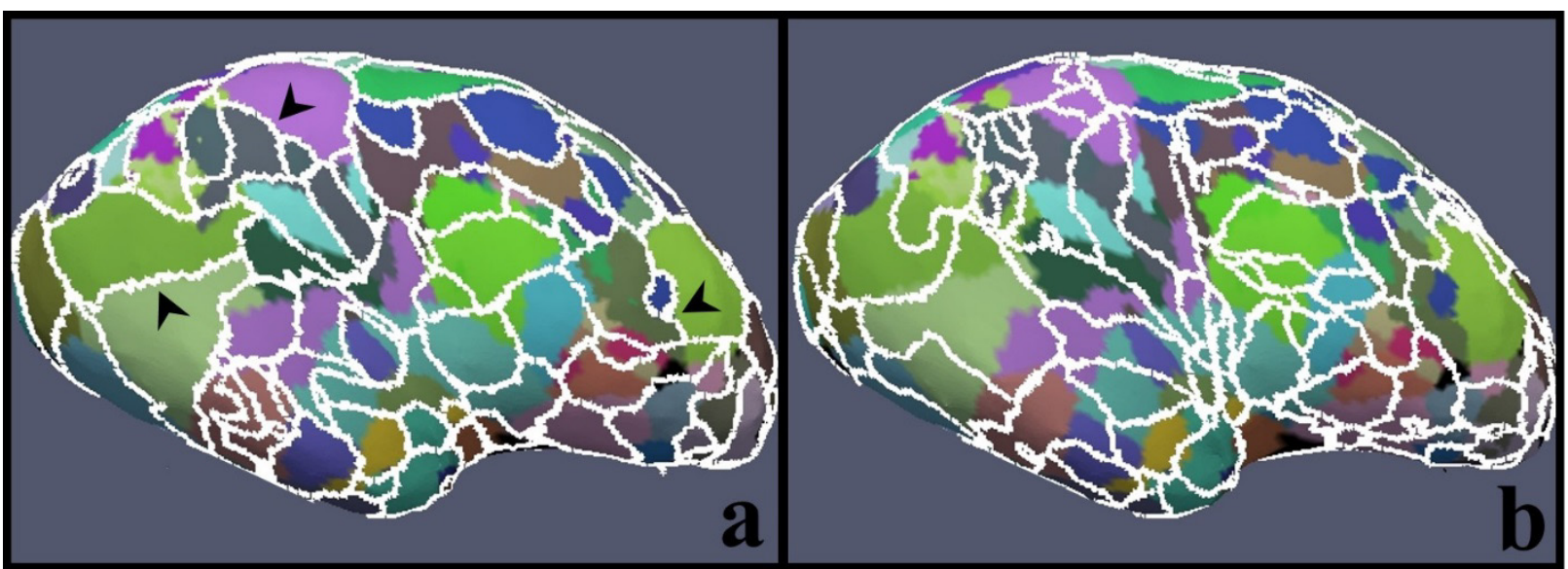

Figure 8.(a) The parcellation boundaries (white curves) of one HCP subject via experiment \#1

3 in strategy \#1 were superimposed to the same HCP subject parcellated via experiment \#2 in

4 strategy \#1; (b) The parcellation boundaries (white curves) of one subject from Dataset 1 were

5 superimposed to the same parcellation in (a) via registration in strategy \#2; Black arrows

6 highlight some matching boundaries.

8 In summary, the full-set HCP dMRIdata derived deterministic fiber tractscan produce

9 parcellation results relatively similar to theconventional DTI data. This preliminary cross-

10 modality comparison results show the promise to conduct an upgraded parcellation framework

11 based on the full-set HCP data. In the future work, to fully taking the advantages of the HCP data,

12 we plan to develop new connectional profile descriptors and look for denser landmarks on the

13 full-set HCP data, which might be helpful to promote the parcellation framework to a higher 14 level.

\section{DISCUSSION}

17 In general, the advantages of our work over other structural connectivity based cortical 18 parcellation methods (e.g., Anwander et al., 2007; Tomassini et al., 2007;Behrens et al., 2008; 
1 Perrin et al., 2008; Beckmann et al., 2009; Roca et al., 2009; Clarkson et al., 2010; Roca et al.,

2 2010; Wang et al., 2013) are three-folds. (1) Many connectivity-based methods need a large

3 group of pre-defined seeds/ROIs to establish the connectivity matrix for vertices/voxels which

4 need to be classified (e.g., Behrens et al., 2008; Perrin et al., 2008; Roca et al., 2010). The main

5 challenge to deal with is the huge dimension. Although different approaches have been proposed

6 to reduce the dimension, it still might be a barrier to prevent those methods from being applied to

7 the entire cortex and across populations; (2) It is difficult to establish correspondences of

8 parcellations across subjects. Some works (e.g., Roca et al., 2009) directly provided a solution by

9 group-wisely clustering the connectivity matrices, while some other methods proposed a solution

10 by integrating connectivity information into registration (e.g., Wang et al., 2013). Those methods,

11 however, may have difficulty in dealing with huge dimension problem or be lacking the capacity

12 of considering and preserving individual variance; (3) It is difficult to automatically determine a

13 suitable cluster number. In contrast, our framework used the trace-map model to encode

14 connectivity profiles so that connectivity parcellation is converted into a parcellation problem

15 based on features, which greatly reduce the dimension. DICCCOLs used in this paper provides

16 not only an individual but also consistent and corresponding reference across subjects and

17 populations, which guarantees the parcellations across subjects to have correspondence.

18 Meantime, based on DICCCOLs correspondence, the group-wise classification algorithm in this

19 paper also allows the vertices on different subjects clustered into the same corresponding parcels

20 in their own way. This can preserve a certain level of individual variance. At last, a fully

21 automatic hierarchical scheme is proposed to provide a spectrum of parcellations. Each finer

22 parcellation level is based on the previous coarser level. That is, the parcels in coarser level are

23 further split into sub-parcels. In each hierarchical level, the algorithm automatically determines 
1 the cluster numbers. Therefore, it is feasible to use this spectrum of parcellations with

2 hierarchical structure. For example, in Figure 7, we superimposed level $2 \& 3$ parcellation levels

3 because they are good enough to match the granularity of the functional clusters. If we overlaid

4 level 5 parcellation, the finer parcellation boundaries will look messy to highlight the boundary

5 consistency. But in Figure 6, ICA and ReHo clusters based on resting state fMRI have higher

6 granularity and the consistency of their borders and parcellation boundaries can be better

7 appreciated in the $5^{\text {th }}$ level. In summary, different structural/functional clusters have different

8 granularity levels. It is more flexible to use a spectrum of parcellation to conduct comparison

9 studies.

11 In the future, the framework can be improved in the following directions. First, at the current 12 stage, our framework takes 4 hours to obtain a group-wise parcellation on the basis of eight 13 subjects using a typical PC (Intel(R) Xeon(R) CPU model; CPU MHz=1600; Memory 50 GB).

14 However, the computation load is an important limitation to overcome in the future to scale the

15 current framework to a larger dataset. Second, conceptually, our framework is of group-wise

16 fashion so that if we include more subjects, the identified consistent parcels will be more

17 commonly present across subject. Meanwhile, including more subjects will also introduce more

18 variability. Therefore, although we are looking for solutions to identify common and consistent

19 parcellations (like those on the frontal lobe in Figure 5(a)), introducing more variability may

20 decrease the group-wise consistency, which should be dealt with in the future. Third, currently,

21 our framework can generate as dense as three hundreds of parcels. However, higher resolution

22 dMRI data (Sotiropoulos et al., 2013) may potentially and fundamentally bring novel insights to

23 finer-granularity parcellations in the future, though we have already demonstrated that common 
1 parcels identified in relatively lower resolution dMRI data (Dataset 1) can be successfully

2 propagated onto the one with high resolution (HCP Dataset). Also, more powerful and

3 descriptive connectional features should be developed consequently based on higher resolution

4 dMRI data to depict more detailed information. Fourth, the current group-wise frameworkcan

5 still be improved in the future. For example, multi-kernel (Blekas, et al., 2012) concepts might be

6 integrated into the group-wise classification algorithm, so that structural patterns from each

7 individual will be dealt with as heterogeneous sources. Also, in a parcel, multiple Gaussian

8 kernels may exist and may be modeled at the same time in the updated classification algorithm.

9 Those improvements may result in more accurate and consistent parcellation when variability

10 within subject and across subjects is considerably taken into consideration. Fifth, application of

11 the method to disease group.Currently, it was not discussed whether the methods in this paper

12 can be applied to diseased brains, though the methods are not ad hoc for normal brains. The

13 parcellation in this paper is based on dMRI-derived white matter structural connectivity, and the

14 fiber bundle shape descriptor, trace-map, was used as a surrogate feature to depict the structural

15 connectivity. Aberrant axonal pathways in disordered brains may lead to dMRI measurements

16 different from controls (e.g., Kubicki et al., 2005; Alexander et al., 2007)). For example,

17 abnormal FA/MD values are found in corpus callosum of autism brains (Alexander et al., 2007).

18 Those abnormal measurements may consequently lead to abnormal structural connectivity

19 profiles, such as fiber bundle shapes. Therefore, it might be risky to obtain parcellation on

20 diseased brains by applying our methods to a combined group of controls and patients. Another

21 possible approach is applying the method on a dataset only consisting of patients with the same

22 brain disease, which will produce more reasonable and specific parcellation results for that

23 disease. Then, comparison between such parcellations with control group may be helpful to 
1 identify biomarker (abnormal parcels) on cortices. Those possibilities will be investigated in our

2 future works.

4 In summary, this paper proposed a novel framework for hierarchical group-wise consistent 5 cortical parcellation based on a group-wise classification algorithmthat integrates spatial

6 constraints and uses DICCCOLs as sample landmarks to generate cluster numbers and initial

7 centers via an adaptive AP algorithm. The effectiveness of the group-wise fashion is

8 demonstrated in comparisons with individual-based version of the framework and other methods

9 like the Ncut and hierarchical clustering. Structural and functional connectivity based on group-

10 wise parcellation is consistent across subjects. Importantly, the hierarchical scheme provides a

11 spectrum of parcellation, which is more flexible to be used in scenarios with different scales. The

12 structure derived parcellation is also compared with fMRI-derived maps, like ReHo maps, ICA

13 clusters and activation maps. The comparison result suggests that the structural parcellation may

14 be used as reference to identify functional correspondence, and the parcel borders have the

15 ability to segregate functional homogeneous regions. The framework will be applied and

16 evaluated in cognitive and clinical neuroscience applications in a similar way as other cortical

17 parcellation approaches were applied in the literature. It will be extensively investigated in the

18 future whether the group-wise consistent fine-granularity cortical parcellation framework would

19 be able to reveal novel neuroscientific insights in such applications.

\section{Acknowledgements}

22 T Liu was supported by the NIH R01 DA-033393, NIH R01 AG-042599, NSF CAREER Award

23 IIS-1149260, and NSF CBET-1302089. L Guo was supported by NSFC 61273362 and 61333017. 
1 T Zhang was supported by supported by NSFC 31500798, the fundamental research funds for

2 the central universities.

4 References

5 Alexander AL, Lee JE, Lazar M, Boudos R, DuBray MB, Oakes TR, Miller JN, Lu J, Jeong EK,

6 McMahon WM, Bigler ED, Lainhart JE. (2007) Diffusion tensor imaging of the corpus callosum

7 in Autism. Neuroimage. 34(1):61-73.

8 Anwander A, Tittgemeyer M, von Cramon DY, Friederici AD, Knösche TR(2007) Connectivity-

9 Based Parcellation of Broca's Area. Cereb Cortex. 17(4):816-25

10 Bastiani M, Shah N, Goebel R, Roebroeck A(2012) Human cortical connectome reconstruction

11 from diffusion weighted MRI: the effect of tractography algorithm. NeuroImage 62 (3): 1732

121749

13 Beckmann C, DeLuca M, Devlin J, Smith S(2005) Investigations into resting-state connectivity

14 using independent component analysis. Phil Trans of Royal Soc. 360: 1001-1013

15 Beckmann M, Johansen-Berg H, Rushworth MF(2009) Connectivity-based parcellation of

16 human cingulate cortex and its relation to functional specialization. J Neurosci. 29(4): 1175-90

17 Behrens TE, Johansen-Berg H, Woolrich MW, Smith SM, Wheeler-Kingshott CA, Boulby

18 PA, Barker GJ, Sillery EL, Sheehan K, Ciccarelli O, Thompson AJ, Brady JM, Matthews

$19 \operatorname{PM}(2003)$ Non-invasive mapping of connections between human thalamus and cortex using

20 diffusion imaging. Nat Neurosci. 6(7):750-7

21 Blekas k, Likas A(2012) The mixture of multi-kernel relevance vector machines model. Data

22 Mining (ICDM). 111-120

23 Blumensath T, Jbabdi S, Glasser MF, Van Essen DC, UgurbilK, Behrens TE, Smith 
1 SM(2013)Spatially constrained hierarchical parcellation of the brain with resting-state fMRI.

2 Neuroimage. 76:313-24

3 Clarkson MJ, Malone IB, Modat M, Leung KK, Ryan N, Alexander DC, Fox NC, Ourselin

4 S(2010) A Framework for using diffusion weighted imaging to improve cortical parcellation.

5 MICCAI 2010. 13(Pt1):534-41

6 Cohen AL, Fair DA, Dosenbach NU, Miezin FM, Dierker D, Van Essen DC, Schlaggar BL,

7 Petersen SE(2008) Defining functional areas in individual human brains using resting functional

8 connectivity MRI. NeuroImage, 41(1):45-57

9 Cordes D, Haughton VM, Arfanakis $\quad$ K, Wendt $\quad$ GJ, Turski PA, Moritz CH, Quigley

10 MA, Meyerand ME(2000) Mapping functionally related regions of brain with functional

11 connectivity MR imaging. AJNR Am J Neuroradiol. 21(9):1636-44

12 Corouge I, Gouttard S, Gerig G(2004) Towards a shape model of white matter fiber bundles

13 using diffusion tensor MRI. Biomedical imaging: nano to macro, 2004: IEEE International

14 Symposium on. IEEE. 344-347

15 Desikan RS, Ségonne F, Fischl B, Quinn, BT, Dickerson BC, Blacker D, Buckner RL, Dale AM,

16 Maguire RP, Hyman BT (2006) An automated labeling system for subdividing the human

17 cerebral cortex on MRI scans into gyral based regions of interest. NeuroImage 31(3): 968-980

18 Dice LR (1945) Measures of the amount of ecologic association between species. Ecology 26 (3):

$19 \quad 297-302$

20 Faraco CC, Unsworth N, Langley J, Terry D, Li K, Zhang D, Liu T, Miller LS(2011) Complex

21 span tasks and hippocampal recruitment during working memory. Neuroimage. 55(2): 77-87

22 Fischl B, Sereno MI, Dale AM(1999) Cortical surface-based analysis II: inflation, flattening, and

23 a surface-based coordinate system. NeuroImage. 9(2):195-207 
1 Fischl B, Van Der Kouwe A, Destrieux C, Halgren E, Ségonne F, Salat, DH, Busa E, Seidman LJ,

2 Goldstein J, Kennedy D(2004) Automatically parcellating the human cerebral cortex. Cereb.

3 Cortex.s 14(1): 11-22

4 Frey BJ and Dueck D(2007) Clustering by Passing Messages between Data Points. Science. $5 \quad 315: 972-976$

6 Gerig G, Gouttard S, Corouge I(2004) Analysis of brain white matter via fiber tract modeling.

7 Engineering in Medicine and Biology Society, 2004: IEMBS'04. $26^{\text {th }}$ Annual International

8 Conference of the IEEE 2:4421-4424

9 Gong G, He Y, Concha L, Lebel C, Gross D, Evans A, Beaulieu C(2009) Mapping anatomical

10 connectivity patterns of human cerebral cortex using in vivo diffusion tensor imaging

11 tractography. Cereb. Cortex. 19(3): 524-536

12 Hagmann P, Kurant M, Gigandet X, Thiran P, Wedeen V, Meuli R, Thiran J(2007) Mapping

13 human whole-brain structural networks with diffusion MRI. PLoS One. 2 (7):597

14 Hampson M, Peterson BS, Skudlarski P, Gatenby JC, Gore JC(2002) Detection of functional

15 connectivity using temporal correlations in MR images. Hum Brain Mapp. 15(4):247-62

16 Honey CJ, Sporns O, Cammoun L, Gigandet X, Thiran JP, Meuli R, Hagmann P(2009)

17 Predicting human resting-state functional connectivity from structural connectivity. PNAS.

18 106(6): 2035-40

19 Jbabdi S, Woolrich MW, Behrens TEJ (2009) Multiple-subjects connectivity-based parcellation

20 using hierarchical Dirichlet process mixture models. NeuroImage. 44(2):373-384.

21 Jbabdi S, Sotiropoulos SN, Savio AM, Graña M, Behrens TE (2012) Model-based analysis of

22 multishell diffusion MR data for tractography: how to get over fitting problems.Magn Reson

23 Med.68(6):1846-55. 
1 Johansen-Berg H, Behrens TE, Robson MD, Drobnjak I, Rushworth MF, Brady JM, Smith SM,

2 Higham DJ, Matthews $\mathrm{PM}(2004)$ Changes in connectivity profiles define functionally distinct

3 regions in human medial frontal cortex. Proc. Natl. Acad. Sci. U.S.A. 101:13335-13340

4 Klein JC, Behrens TE, Robson MD, Mackay CE, Higham DJ, Johansen-Berg H(2007)

5 Connectivity-based parcellation of human cortex using diffusion MRI: establishing

6 reproducibility, validity and observer independence in Ba 44/45 and SMA/pre-sma. NeuroImage

$7 \quad 34(1): 204-211$

8 Kubicki M, Park H, Westin CF, Nestor PG, Mulkern RV, Maier SE, Niznikiewicz M, Connor EE,

9 Levitt JJ, Frumin M, Kikinis R, Jolesz FA, McCarley RW, Shenton ME. (2005) DTI and MTR

10 abnormalities in schizophrenia: Analysis of white matter integrity. Neuroimage.26(4): 1109 -

111118.

12 Li K, Guo L, Li G, Nie J, Faraco C, Cui G, Zhao Q, Miller LS, Liu T(2010a) Gyral folding 13 pattern analysis via surface profiling. 52(4):1202-14

14 Li K, Guo L, Zhu D, Hu X, Han J, Liu T(2010b) Individualized ROI optimization via 15 maximization of group-wise consistency of structural and functional profiles. NIPS. 23

16 Liu T, Li H, Wong K, Tarokh A, GuoL, Wong STC(2007) Brain tissue segmentation based on

17 DTI data. NeuroImage. 38(1):114-123

18 Liu T, Nie J, Tarokh A, Guo L, Wong STC(2008) Reconstruction of central cortical surface from 19 brain MRI images: Method and application. NeuroImage. 40(3):991-1002

20 Liu Y, Wang $\quad \mathrm{K}$, Yu $\quad$ C, He $\quad$ Y, Zhou $\quad$ Y, Liang $\quad$ M, Wang $\quad$ L, Jiang $\quad \mathrm{T}(2008)$

21 Regional homogeneity, functional connectivity and imaging markers of Alzheimer's disease: a

22 review of resting-state fMRI studies. Neuropsychologia. 46(6):1648-56

23 Lohmann G, von Cramon DY(2000) Automatic labelling of the human cortical surface using 
1 sulcal basins. Med Image Anal. 4(3):179-88Nelson SM, Cohen AL, Power JD, Wig GS, Miezin

2 FM, Wheeler ME, Velanova K, Donaldson DI, Phillips JS, Schlaggar BL, Petersen SE(2010) A

3 parcellation scheme for human left lateral parietal cortex. Neuron. 67(1):156-170

4 O'Donnell LJ, Golby AJ, Westin CF(2013) Neuroimage. Fiber clustering versus the parcellation-

5 based connectome. 80:283-9.

6 Passingham RE, Stephan KE, Kötter R(2002) The anatomical basis of functional localization in

7 the cortex, Nat Rev Neurosci. 3(8):606-16

8 Perrin M, Cointepas Y, Cachia A, Poupon C, Thirion B, Rivière D, Cathier P, El Kouby

9 V, Constantinesco A, Le Bihan D, Mangin JF(2008) Connectivity-based parcellation of the

10 cortical mantle using q-ball diffusion imaging. Int J Biomed Imaging. 2008:368406

11 Postelnicu GM, Zöllei L, Fischl B. (2009) Combined Volumetric and Surface Registration. IEEE

12 Transactions on Medical Imaging. 28 (4):508-522.

13 Rettmann ME, Han X, Xu C, Prince JL(2002) Automated sulcal segmentation using watersheds

14 on the cortical surface.NeuroImage.15(2):329-44.

15 Shen X, Tokoglu F, Papademetris X, Constable RT(2013) Groupwise whole-

16 brain parcellation from resting-state fMRI data for network node identification. Neuroimage. 82 :

$17 \quad 403-15$

18 Roca P, Rivière D, Guevara P, Poupon C, Mangin JF(2009) Tractography-based parcellation of

19 the cortex using a spatially-informed dimension reduction of the connectivitymatrix. Med Image

20 Comput Comput Assist Interv. 12(Pt 1):935-42

21 Roca P, Tucholka A, Rivière $\quad$ D, Guevara $\quad$ P, Poupon $\quad$ C, Mangin JF(2010) Inter-

22 subject connectivity-based parcellation of a patch of cerebral cortex. Med Image Comput

23 Comput Assist Interv. 13(Pt 2):347-54 
1 Shen D, Davatzikos C(2002) HAMMER: hierarchical attribute matching mechanism for elastic

2 registration. IEEE Trans Med Imaging. 21(11): 1421-39

3 Smith SM, Beckmann CF, Andersson J, Auerbach EJ, Bijsterbosch J, Douaud G, Duff

4 E, Feinberg DA, Griffanti L, Harms MP, Kelly M, Laumann T, Miller KL, Moeller S, Petersen

5 S, Power J, Salimi-Khorshidi G, Snyder AZ, Vu AT, Woolrich MW, Xu J, Yacoub E, Uğurbil

6 K, Van Essen DC, Glasser MF WU-Minn HCP Consortium(2013) Resting-state fMRI in the

7 Human Connectome Project. Neuroimage. 80:144-68

8 Sotiropoulos, SN, Jbabdi S, Xu J, Andersson JL, Moeller S, Auerbach EJ, Glasser MF,

9 Hernandez M, Sapiro G, Jenkinson M, Feinberg DA, Yacoub E, Lenglet C, Van Essen DC,

10 Ugurbil K, Behrens TE(2013) Advances in diffusion MRI acquisition and processing in the

11 Human Connectome Project. Neuroimage. 80:125-43

12 Thompson P, Toga AW (1996) A surface-based technique for warping three-dimensional images

13 of the brain. IEEE Trans Med Imaging. 15(4):402-17

14 Tomassini V, Jbabdi S, Klein JC, Behrens TE, Pozzilli C, Matthews PM, Rushworth MF,

15 Johansen-Berg H(2007) Diffusion-weighted imaging tractography-based parcellation of the

16 human lateral premotor cortex identifies dorsal and ventral subregions with anatomical and

17 functional specializations. J. Neurosci. 27 (38):10259-10269.

18 Van Essen DC, Dierker DL(2007) Surface-based and probabilistic atlases of primate cerebral

19 cortex. Neuron. 56(2):209-25

20 van den Heuvel M, Mandl R, Hulshoff Pol H(2008) Normalized cut group clustering of resting-

21 state fMRI data. PLoS One. 3(4):e2001

22 Von Luxburg U. (2007) A tutorial on spectral clustering. Statistics and Computing. 17(4):395$23 \quad 416$ 
1 Wang KJ, Zhang JY, Li D, Zhang XN, Guo T (2007) Adaptive Affinity Propagation Clustering.

2 Acta Automatica Sinica,33(12):1242-1246

3 Wang Q, Yap PT, Wu G, Shen D(2013) Diffusion Tensor Image Registration Using Hybrid

4 Connectivity and Tensor Features. Human Brain Mapping. Accepted.

5 Ward JHJ(1963) Hierarchical grouping to optimize an objective function. J. Am. Stat. Assoc.

$6 \quad 58: 236-244$

7 Yang F, Kruggel F(2008) Automatic segmentation of human brain sulci. Med Image Anal.

$8 \quad 12(4): 442-51$

9 Yeh FC, Verstynen TD, Wang Y, Fernández-Miranda JC, Tseng WY (2013) Deterministic

10 diffusion fiber tracking improved by quantitative anisotropy. PLoS One. 8(11): 380713

11 Zang Y, Jiang T, Lu Y, He Y, Tian L(2004) Regional homogeneity approach to fMRI data

12 analysis. Neuroimage. 22(1):394-400

13 Zhang Y, Brady M, Smith S(2001) Segmentation of brain MR images through a hidden Markov

14 random field model and the expectation maximization algorithm. IEEE Trans Med Imaging.

$15 \quad 20: 45-57$

16 Zhang T, Guo L, Li G, Nie J, Liu T(2009) Parametric Representation of Cortical Surface Folding

17 based on Polynomials. Medical Image Computing and Computer Assisted Intervention

18 (MICCAI). 12(Pt 2):184-91

19 Zhang D, Guo L, Li G, Nie J, Deng F, Li K, Hu X, Zhang T, Jiang X, Zhu D, Zhao Q, Liu

$20 \mathrm{~T}(2010)$ Automatic cortical surface parcellation based on fiber density information. 2010 IEEE

21 International Symposium on Biomedical Imaging. 1133-1136

22 Zhu D, Li K, Faraco CC, Deng F, Zhang $\quad$ D, Guo $\quad$ L, Miller $\quad$ LS, Liu $\quad$ T(2012a)

23 Optimization of functional brain ROIs via maximization of consistency of structural connectivity 
1 profiles. Neuroimage. 59(2):1382-93

2 Zhu D, Li K, Guo L, Jiang X, Zhang T, Zhang D, Chen H, Deng F, Faraco C, Jin C, Wee CY,

3 Yuan Y, Lv P, Yin Y, Hu X, Duan L, Hu X, Han J, Wang L, Shen D, Miller LS, Li L, Liu T(2012b)

4 DICCCOL: dense individualized and common connectivity-based cortical landmarks. Cereb

5 Cortex. 23(4):786-800

6 Zöllei L, Stevens A, Huber K, Kakunoori S, Fischl B. (2010) Improved Tractography Alignment

7 Using Combined Volumetric and Surface Registration. NeuroImage 51(1):206-213 


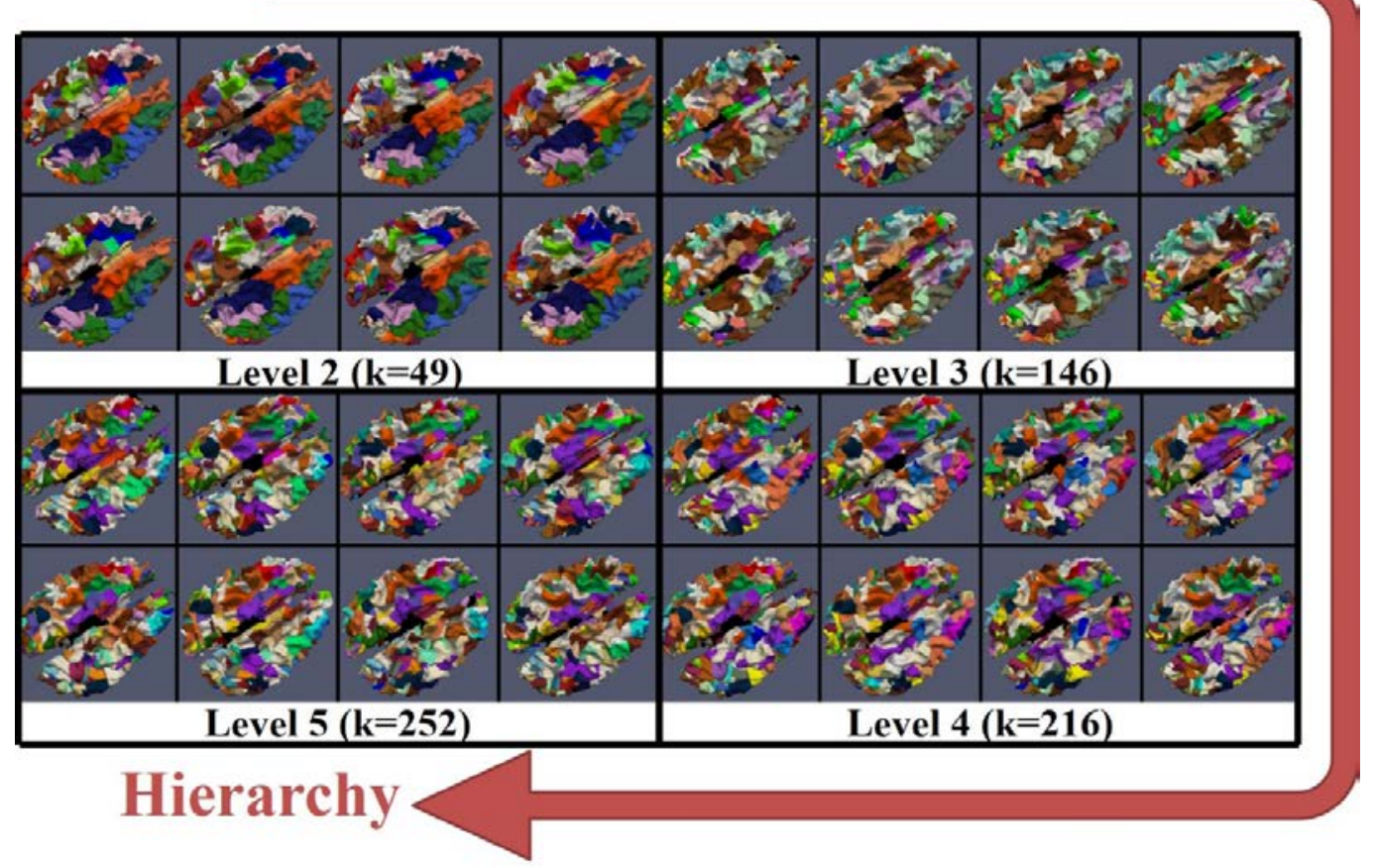

